\title{
A Conditional Fourier-Feynman Transform and Conditional Convolution Product with Change of Scales on a Function Space II
}

\author{
Dong Hyun Cho \\ Department of Mathematics, Kyonggi University, Suwon 16227, Republic of Korea \\ Correspondence should be addressed to Dong Hyun Cho; j94385@kyonggi.ac.kr
}

Received 29 July 2016; Accepted 24 November 2016; Published 28 February 2017

Academic Editor: Ramón M. Rodríguez-Dagnino

Copyright (C) 2017 Dong Hyun Cho. This is an open access article distributed under the Creative Commons Attribution License, which permits unrestricted use, distribution, and reproduction in any medium, provided the original work is properly cited.

Using a simple formula for conditional expectations over continuous paths, we will evaluate conditional expectations which are types of analytic conditional Fourier-Feynman transforms and conditional convolution products of generalized cylinder functions and the functions in a Banach algebra which is the space of generalized Fourier transforms of the measures on the Borel class of $L_{2}[0, T]$. We will then investigate their relationships. Particularly, we prove that the conditional transform of the conditional convolution product can be expressed by the product of the conditional transforms of each function. Finally we will establish change of scale formulas for the conditional transforms and the conditional convolution products. In these evaluation formulas and change of scale formulas, we use multivariate normal distributions so that the conditioning function does not contain present positions of the paths.

\section{Introduction}

Let $C[0, T]$ denote an analogue of Wiener space which is the space of real-valued continuous functions on the interval $[0, T][1]$. The conditional expectations which are types of analytic conditional Fourier-Feynman transforms and conditional convolution products on $C[0, T]$ are introduced by the author [2] using a conditioning function which contains present positions of the paths. According to the author's paper, he investigated the effects that drift has on the conditional Fourier-Feynman transform, the conditional convolution product, and various relationships that occur among them. In particular, he derived several change of scale formulas for the conditional transforms and the conditional convolution products, which simplify evaluating the conditional expectations, because the probability measure used on $C[0, T]$ cannot be scale-invariant $[3,4]$.

Let $a$ be absolutely continuous on $[0, T]$ and let $h$ be of bounded variation with $h \neq 0$ a.e. on $[0, T]$. Define a stochastic process $Z: C[0, T] \times[0, T] \rightarrow \mathbb{R}$ by

$$
Z(x, t)=\left(h \chi_{[0, t]}, x\right)+x(0)+a(t),
$$

for $x \in C[0, T]$ and $t \in[0, T]$, where $(\cdot, \cdot)$ denotes the PaleyWiener-Zygmund stochastic integral [1]. For a partition $0=$ $t_{0}<t_{1}<\cdots<t_{n}<t_{n+1}=T$ of $[0, T]$, define random vectors $Z_{n}: C[0, T] \rightarrow \mathbb{R}^{n+1}$ and $Z_{n+1}: C[0, T] \rightarrow \mathbb{R}^{n+2}$ by

$$
\begin{aligned}
& Z_{n}(x)=\left(Z\left(x, t_{0}\right), Z\left(x, t_{1}\right), \ldots, Z\left(x, t_{n}\right)\right), \\
& Z_{n+1}(x) \\
& \quad=\left(Z\left(x, t_{0}\right), Z\left(x, t_{1}\right), \ldots, Z\left(x, t_{n}\right), Z\left(x, t_{n+1}\right)\right) .
\end{aligned}
$$

With the conditioning function $Z_{n+1}$ which contains the present position $Z(x, T)$ of the path $Z(x, \cdot)$, the author [5] evaluated the conditional Fourier-Feynman transforms and the conditional convolution products of the functions given by

$$
\begin{aligned}
& \int_{L_{2}[0, T]} \exp \{i(v, Z(x, \cdot))\} d \sigma(v), \\
& \quad f\left(\left(v_{1}, Z(x, \cdot)\right), \ldots,\left(v_{r}, Z(x, \cdot)\right)\right),
\end{aligned}
$$

where $\sigma$ is a complex Borel measure of bounded variation on $L_{2}[0, T], f \in L_{p}\left(\mathbb{R}^{r}\right)$ with $1 \leq p \leq \infty$, and 
$\left\{v_{1}, \ldots, v_{r}\right\}$ is an orthonormal subset of $L_{2}[0, T]$. He then investigated relationships between the conditional FourierFeynman transforms and the conditional convolution products of the functions given by (3).

In this paper, using a simple formula for conditional expectations over continuous paths [6], we evaluate conditional expectations of generalized cylinder functions and the functions in a Banach algebra which plays significant roles in Feynman integration theories and quantum mechanics. We then investigate their relationships. Particularly, we prove that the conditional transform of the convolution product can be expressed by the product of the conditional transforms of each function. Finally we establish change of scale formulas for the conditional expectations. In these evaluation formulas and change of scale formulas we use multivariate normal distributions so that the conditioning function does not contain the present positions of the paths. In fact, with the conditioning function $Z_{n}$ which does not contain the present position $Z(x, T)$ of the path $Z(x, \cdot)$, we evaluate conditional expectations, that is, the conditional Fourier-Feynman transforms and the conditional convolution products of the functions given by (3). We then show that the $L_{p}$-analytic conditional Fourier-Feynman transform $T_{q}^{(p)}\left[\left[\left(F_{Z} * G_{Z}\right)_{q}\right.\right.$ । $\left.\left.Z_{n}\right]\left(\cdot, \vec{\xi}_{n}\right) \mid Z_{n}\right]$ of the conditional convolution product for the functions $F_{Z}$ and $G_{Z}$ which are described in (3) can be expressed by the formula

$$
\begin{aligned}
T_{q}^{(p)} & {\left[\left[\left(F_{Z} * G_{Z}\right)_{q} \mid Z_{n}\right]\left(\cdot, \vec{\xi}_{n}\right) \mid Z_{n}\right]\left(y, \vec{\eta}_{n}\right) } \\
= & {\left[T _ { q } ^ { ( p ) } [ F _ { Z } | Z _ { n } ] \left(\frac{1}{\sqrt{2}} y\right.\right.} \\
+ & \left.\left.(\sqrt{2}-1) a, \frac{1}{\sqrt{2}}\left(\vec{\eta}_{n}+\vec{\xi}_{n}\right)-(\sqrt{2}-1) \vec{a}_{n}\right)\right] \\
& \cdot\left[T_{q}^{(p)}\left[G_{Z} \mid Z_{n}\right]\left(\frac{1}{\sqrt{2}} y-a, \frac{1}{\sqrt{2}}\left(\vec{\eta}_{n}-\vec{\xi}_{n}\right)+\vec{a}_{n}\right)\right],
\end{aligned}
$$

for a nonzero real $q$, almost surely $y \in C[0, T]$, and $P_{Z_{n}}$ almost surely $\vec{\xi}_{n}, \vec{\eta}_{n} \in \mathbb{R}^{n+1}$, where $\vec{a}_{n}=\left(a\left(t_{0}\right), a\left(t_{1}\right), \ldots, a\left(t_{n}\right)\right)$ and $P_{Z_{n}}$ is the probability distribution of $Z_{n}$ on the Borel class of $\mathbb{R}^{n+1}$. Compared with the results in $[5,7]$, the conditioning function $Z_{n}$ in this paper does not contain the present position $Z(x, T)$ of $Z(x, \cdot)$ and the effects of drift $a$ depend on the polygonal function of $a$ so that we can generalize the theorems in $[5,7]$ and the results of this paper do not depend on a particular choice of the initial distribution of the paths.

\section{Preliminaries on an Analogue of Wiener Space}

We begin this section with introducing a concrete form of an analogue of Wiener space which is our underlying space.

For a positive real $T$ let $C[0, T]$ denote the space of realvalued continuous functions on the time interval $[0, T]$ with the supremum norm. For $\vec{t}=\left(t_{0}, t_{1}, \ldots, t_{n}\right)$ with $0=t_{0}<t_{1}<$ $\cdots<t_{n} \leq T$ let $J_{\vec{t}}: C[0, T] \rightarrow \mathbb{R}^{n+1}$ be the function given by $J_{\vec{t}}(x)=\left(x\left(t_{0}\right), x\left(t_{1}\right), \ldots, x\left(t_{n}\right)\right)$. For $B_{j}(j=0,1, \ldots, n)$ in the
Borel class $\mathscr{B}(\mathbb{R})$ of $\mathbb{R}$, the subset $J_{\vec{t}}^{-1}\left(\prod_{j=0}^{n} B_{j}\right)$ of $C[0, T]$ is called an interval and let $\mathscr{I}$ be the set of all such intervals. For a probability measure $\varphi$ on $\mathscr{B}(\mathbb{R})$, let

$$
\begin{gathered}
m_{\varphi}\left[J_{\vec{t}}^{-1}\left(\prod_{j=0}^{n} B_{j}\right)\right]=\left[\prod_{j=1}^{n} \frac{1}{2 \pi\left(t_{j}-t_{j-1}\right)}\right]^{1 / 2} \\
\cdot \int_{B_{0}} \int_{\prod_{j=1}^{n} B_{j}} W_{n}\left(\vec{t}, \vec{u}, u_{0}\right) d \vec{u} d \varphi\left(u_{0}\right),
\end{gathered}
$$

where $W\left(\vec{t}, \vec{u}, u_{0}\right)=\exp \left\{-(1 / 2) \sum_{j=1}^{n}\left(\left(u_{j}-u_{j-1}\right)^{2} /\left(t_{j}-t_{j-1}\right)\right)\right\}$ for $\vec{u}=\left(u_{1}, \ldots, u_{n}\right) . \mathscr{B}(C[0, T])$, the Borel $\sigma$-algebra of $C[0, T]$, coincides with the smallest $\sigma$-algebra generated by $\mathscr{I}$ and there exists a unique probability measure $w_{\varphi}$ on $C[0, T]$ such that $w_{\varphi}(I)=m_{\varphi}(I)$ for all $I \in \mathscr{I}$. This measure $w_{\varphi}$ is called an analogue of Wiener measure associated with the probability measure $\varphi[1]$.

Let $\mathbb{C}$ and $\mathbb{C}_{+}$denote the sets of complex numbers and complex numbers with positive real parts, respectively. Let $F: C[0, T] \rightarrow \mathbb{C}$ be integrable and let $X_{\tau}$ be a random vector on $C[0, T]$ assuming that the value space of $X_{\tau}$ is a normed space with the Borel $\sigma$-algebra. Then we have the conditional expectation $E\left[F \mid X_{\tau}\right]$ of $F$ given $X_{\tau}$ from a wellknown probability theory [8]. Furthermore, there exists a $P_{X_{\tau}}$ integrable complex-valued function $\phi$ on the value space of $X_{\tau}$ such that

$$
E\left[F \mid X_{\tau}\right](x)=\left(\phi \circ X_{\tau}\right)(x)
$$

for $w_{\varphi}$ a.e. $x \in C[0, T]$,

where $P_{X_{\tau}}$ is the probability distribution of $X_{\tau}$. The function $\phi$ is called the conditional $w_{\varphi}$-integral of $F$ given $X_{\tau}$ and it is also denoted by $E\left[F \mid X_{\tau}\right]$.

For an extended real number $p$ with $1<p \leq \infty$, suppose that $p$ and $p^{\prime}$ are related by $1 / p+1 / p^{\prime}=1$ (possibly $p^{\prime}=1$ if $p=\infty)$. Let $q \in \mathbb{R}-\{0\}$. For $\lambda \in \mathbb{C}_{+}$let $F_{\lambda}$ be a measurable function on $C[0, T]$ such that

$$
\lim _{\lambda \rightarrow-i q} E\left[\left|F_{\lambda}-F\right|^{p^{\prime}}\right]=0 \text {. }
$$

Then we write

$$
\lim _{\lambda \rightarrow-i q}\left(w^{p^{\prime}}\right)\left(F_{\lambda}\right)=F
$$

Let $0=t_{0}<t_{1}<\cdots<t_{n}<t_{n+1}=T$ be a partition of $[0, T]$, where $n$ is a fixed nonnegative integer. Let $h$ be of bounded variation with $h \neq 0$ a.e. on $[0, T]$. Let $a$ be absolutely continuous on $[0, T]$ and define stochastic processes $X, Z: C[0, T] \times[0, T] \rightarrow \mathbb{R}$ by

$$
\begin{aligned}
& X(x, t)=\left(h \chi_{[0, t]}, x\right), \\
& Z(x, t)=X(x, t)+x(0)+a(t),
\end{aligned}
$$

for $x \in C[0, T]$ and $t \in[0, T]$, where $\chi$ denotes an indicator function. Define a random vector $Z_{n}: C[0, T] \rightarrow \mathbb{R}^{n+1}$ by

$$
Z_{n}(x)=\left(Z\left(x, t_{0}\right), Z\left(x, t_{1}\right), \ldots, Z\left(x, t_{n}\right)\right),
$$


for $x \in C[0, T]$. For $t \in[0, T]$, let $b(t)=\int_{0}^{t}[h(s)]^{2} d s$ and for any function $f$ on $[0, T]$, define a polygonal function $P_{b, n+1}(f)$ of $f$ by

$$
\begin{aligned}
& P_{b, n+1}(f)(t)=\sum_{j=1}^{n+1}\left[\frac{b\left(t_{j}\right)-b(t)}{b\left(t_{j}\right)-b\left(t_{j-1}\right)} f\left(t_{j-1}\right)\right. \\
& \left.+\frac{b(t)-b\left(t_{j-1}\right)}{b\left(t_{j}\right)-b\left(t_{j-1}\right)} f\left(t_{j}\right)\right] \chi_{\left(t_{j-1}, t_{j}\right]}(t)+f(0) \chi_{\{0\}}(t),
\end{aligned}
$$

for $t \in[0, T]$. For $\vec{\xi}_{n+1}=\left(\xi_{0}, \xi_{1}, \ldots, \xi_{n}, \xi_{n+1}\right) \in \mathbb{R}^{n+2}$, define a polygonal function $P_{b, n+1}\left(\vec{\xi}_{n+1}\right)$ of $\vec{\xi}_{n+1}$ by (11), where $f\left(t_{j}\right)$ is replaced by $\xi_{j}$. If $\vec{\xi}_{n}=\left(\xi_{0}, \xi_{1}, \ldots, \xi_{n}\right) \in \mathbb{R}^{n+1}, P_{b, n}\left(\vec{\xi}_{n}\right)$ is interpreted as $P_{b, n+1}\left(\vec{\xi}_{n+1}\right)$ with $\xi_{n+1}=\xi_{n}$. For $x \in C[0, T]$ and $t \in[0, T]$ let

$$
\begin{aligned}
A(t) & =a(t)-P_{b, n+1}(a)(t), \\
X_{b, n+1}(x, t) & =X(x, t)-P_{b, n+1}(X(x, \cdot))(t), \\
Z_{b, n+1}(x, t) & =Z(x, t)-P_{b, n+1}(Z(x, \cdot))(t) .
\end{aligned}
$$

For $\vec{a}, \vec{u} \in \mathbb{R}^{r}, \lambda \in \mathbb{C}$, and any nonsingular positive $r \times r$ matrix $A_{r}$ on $\mathbb{R}$, let

$$
\begin{aligned}
& \Psi_{r}\left(\lambda, \vec{a}, A_{r}, \vec{u}\right)=\left[\frac{\lambda^{r}}{(2 \pi)^{r}\left|A_{r}\right|}\right]^{1 / 2} \\
& \cdot \exp \left\{-\frac{\lambda}{2}\left\langle A_{r}^{-1}(\vec{u}-\vec{a}), \vec{u}-\vec{a}\right\rangle_{\mathbb{R}}\right\},
\end{aligned}
$$

where $\langle\cdot, \cdot\rangle_{\mathbb{R}}$ denotes the dot product on $\mathbb{R}^{r}$. Let $I_{r}$ be the $r \times r$ identity matrix on $\mathbb{R}$. For a measurable function $F$ : $C[0, T] \rightarrow \mathbb{C}$, let

$$
F_{Z}(x, y)=F(Z(x, \cdot)+y) \quad \text { for } x, y \in C[0, T],
$$

let $F_{Z}^{\lambda}(x, y)=F_{Z}\left(\lambda^{-1 / 2} x, y\right)$, and let $Z_{n}^{\lambda}(x)=Z_{n}\left(\lambda^{-1 / 2} x\right)$ for $\lambda>0$. Suppose that $E\left[F_{Z}^{\lambda}(\cdot, y)\right]$ exists, where the expectation is taken over the first variable. By Theorem 2 in [2] and Theorem 7 in [6]

$$
\begin{aligned}
E & {\left[F_{Z}^{\lambda}(\cdot, y) \mid Z_{n}^{\lambda}\right]\left(\vec{\xi}_{n}\right)=\int_{\mathbb{R}} \Psi_{1}\left(\lambda, a(T)-a\left(t_{n}\right), b(T)\right.} \\
& \left.-b\left(t_{n}\right), \xi_{n+1}-\xi_{n}\right) E\left[F \left(y+\lambda^{-1 / 2} X_{b, n+1}(x, \cdot)+A\right.\right. \\
& \left.\left.+P_{b, n+1}\left(\vec{\xi}_{n+1}\right)\right)\right] d \xi_{n+1},
\end{aligned}
$$

for $P_{Z_{n}^{\lambda}}$ a.e. $\vec{\xi}_{n}=\left(\xi_{0}, \xi_{1}, \ldots, \xi_{n}\right) \in \mathbb{R}^{n+1}$, where $\vec{\xi}_{n+1}=\left(\xi_{0}, \xi_{1}\right.$, $\left.\ldots, \xi_{n}, \xi_{n+1}\right), P_{Z_{n}^{\lambda}}$ is the probability distribution of $Z_{n}^{\lambda}$ on $\left(\mathbb{R}^{n+1}, \mathscr{B}\left(\mathbb{R}^{n+1}\right)\right)$ and the expectation is taken over the variable $x$. Let $I_{F_{Z}}^{\lambda}\left(y, \vec{\xi}_{n}\right)$ be the right-hand side of (15). If, for $w_{\varphi}$ a.e. $y \in C[0, T]$ and $P_{Z_{n}}$ a.e. $\vec{\xi}_{n} \in \mathbb{R}^{n+1}, I_{F_{Z}}^{\lambda}\left(y, \vec{\xi}_{n}\right)$ has an analytic extension $J_{\lambda}^{*}\left(F_{Z}\right)\left(y, \vec{\xi}_{n}\right)$ on $\mathbb{C}_{+}$, then it is called a generalized analytic conditional Fourier-Wiener transform of $F$ given $Z_{n}$ with the parameter $\lambda$ and is denoted by

$$
T_{\lambda}\left[F_{Z} \mid Z_{n}\right]\left(y, \vec{\xi}_{n}\right)=J_{\lambda}^{*}\left(F_{Z}\right)\left(y, \vec{\xi}_{n}\right) .
$$

Moreover if $T_{\lambda}\left[F_{Z} \mid Z_{n}\right]\left(y, \vec{\xi}_{n}\right)$ has a limit as $\lambda$ approaches -iq through $\mathbb{C}_{+}$, then it is called a generalized $L_{1}$-analytic conditional Fourier-Feynman transform of $F$ given $Z_{n}$ with the parameter $q$ and is denoted by

$$
T_{q}^{(1)}\left[F_{Z} \mid Z_{n}\right]\left(y, \vec{\xi}_{n}\right)=\lim _{\lambda \rightarrow-i q} T_{\lambda}\left[F_{Z} \mid Z_{n}\right]\left(y, \vec{\xi}_{n}\right) .
$$

For $1<p \leq \infty$, define a generalized $L_{p}$-analytic conditional Fourier-Feynman transform $T_{q}^{(p)}\left[F_{Z} \mid Z_{n}\right]$ of $F$ given $Z_{n}$ by the formula

$$
\begin{aligned}
T_{q}^{(p)} & {\left[F_{Z} \mid Z_{n}\right]\left(\cdot, \vec{\xi}_{n}\right) } \\
& =\lim _{\lambda \rightarrow-i q}\left(w^{p^{\prime}}\right)\left(T_{\lambda}\left[F_{Z} \mid Z_{n}\right]\left(\cdot, \vec{\xi}_{n}\right)\right) \quad \text { (if exists). }
\end{aligned}
$$

Let $G$ be defined on $C[0, T]$. For $\lambda>0$ and $y \in C[0, T]$, redefine $F_{Z}^{\lambda}(\cdot / \sqrt{2}, y / \sqrt{2}) G_{Z}^{\lambda}(-\cdot / \sqrt{2}, y / \sqrt{2})=F_{Z / \sqrt{2}}^{\lambda}(\cdot, y /$ $\sqrt{2}) G_{-Z / \sqrt{2}}^{\lambda}(\cdot, y / \sqrt{2})$ and suppose that $E\left[F_{Z}^{\lambda}(\cdot / \sqrt{2}, y / \sqrt{2}) G_{Z}^{\lambda}(-\cdot /\right.$ $\sqrt{2}, y / \sqrt{2})$ ] exists. By Theorem 2 in [2] and Theorem 7 in [6]

$$
\begin{aligned}
E & {\left[F_{Z}^{\lambda}\left(\frac{\cdot}{\sqrt{2}}, \frac{y}{\sqrt{2}}\right) G_{Z}^{\lambda}\left(-\frac{\dot{1}}{\sqrt{2}}, \frac{y}{\sqrt{2}}\right) \mid Z_{n}^{\lambda}\right]\left(\vec{\xi}_{n}\right)=\int_{\mathbb{R}} \Psi_{1}\left(\lambda, a(T)-a\left(t_{n}\right), b(T)-b\left(t_{n}\right), \xi_{n+1}-\xi_{n}\right) } \\
\cdot & E\left[F\left(\frac{1}{\sqrt{2}}\left[y+\lambda^{-1 / 2} X_{b, n+1}(x, \cdot)+A+P_{b, n+1}\left(\vec{\xi}_{n+1}\right)\right]\right)\right. \\
\cdot & \left.G\left(\frac{1}{\sqrt{2}}\left[y-\lambda^{-1 / 2} X_{b, n+1}(x, \cdot)-A-P_{b, n+1}\left(\vec{\xi}_{n+1}\right)\right]\right)\right] d \xi_{n+1} \cdot
\end{aligned}
$$

Let $K_{F_{Z}, G_{Z}}^{\lambda}\left(y, \vec{\xi}_{n}\right)$ be the right-hand side of (19). If $K_{F_{Z}, G_{Z}}^{\lambda}\left(y, \vec{\xi}_{n}\right)$ has an analytic extension $J_{\lambda}^{*}\left(F_{Z}, G_{Z}\right)\left(y, \vec{\xi}_{n}\right)$ on $\mathbb{C}_{+}$, then it is called a generalized conditional convolution product of $F$ and $G$ given $Z_{n}$ with the parameter $\lambda$ and denoted by

$$
\left[\left(F_{Z} * G_{Z}\right)_{\lambda} \mid Z_{n}\right]\left(y, \vec{\xi}_{n}\right)=J_{\lambda}^{*}\left(F_{Z}, G_{Z}\right)\left(y, \vec{\xi}_{n}\right) .
$$

Moreover if $\left[\left(F_{Z} * G_{Z}\right)_{\lambda} \mid Z_{n}\right]\left(y, \vec{\xi}_{n}\right)$ has a limit as $\lambda$ approaches $-i q$ through $\mathbb{C}_{+}$, then it is called a generalized 
conditional convolution product of $F$ and $G$ given $Z_{n}$ with the parameter $q$ and denoted by

$$
\begin{aligned}
& {\left[\left(F_{Z} * G_{Z}\right)_{q} \mid Z_{n}\right]\left(y, \vec{\xi}_{n}\right)} \\
& \quad=\lim _{\lambda \rightarrow-i q}\left[\left(F_{Z} * G_{Z}\right)_{\lambda} \mid Z_{n}\right]\left(y, \vec{\xi}_{n}\right) .
\end{aligned}
$$

For $j=1, \ldots, n+1$, let

$$
\alpha_{j}=\frac{h}{\sqrt{b\left(t_{j}\right)-b\left(t_{j-1}\right)}} \chi_{\left(t_{j-1}, t_{j}\right]},
$$

let $V$ be the subspace of $L_{2}[0, T]$ generated by $\left\{\alpha_{1}, \ldots\right.$, $\left.\alpha_{n}, \alpha_{n+1}\right\}$, and let $V^{\perp}$ be the orthogonal complement of $V$. Let $\mathscr{P}: L_{2}[0, T] \rightarrow V$ be the orthogonal projection given by

$$
\mathscr{P} v=\sum_{j=1}^{n+1}\left\langle v, \alpha_{j}\right\rangle \alpha_{j}
$$

and let $\mathscr{P}^{\perp}: L_{2}[0, T] \rightarrow V^{\perp}$ be the orthogonal projection. For $\vec{u} \in \mathbb{R}^{r}, \lambda \in \mathbb{C}, \vec{v}=\left(v_{1}, \ldots, v_{r}\right) \in L_{2}^{n}[0, T]$ and any nonsingular positive $r \times r$ matrix $A_{r}$ on $\mathbb{R}$, let

$$
\begin{aligned}
& \left\langle\vec{v} \alpha_{n+1}, \alpha_{n+1}\right\rangle=\left(\left\langle v_{1} \alpha_{n+1}, \alpha_{n+1}\right\rangle, \ldots,\left\langle v_{r} \alpha_{n+1}, \alpha_{n+1}\right\rangle\right), \\
& \Lambda\left(A_{r}\right)=1+\left[b(T)-b\left(t_{n}\right)\right]\left\|A_{r}^{-1}\left\langle\vec{v} \alpha_{n+1}, \alpha_{n+1}\right\rangle\right\|_{\mathbb{R}}^{2}, \\
& \Phi_{r}\left(\lambda, \vec{v}, A_{r}, \vec{u}\right)=\left[\frac{1}{\Lambda\left(A_{r}\right)}\right]^{1 / 2} \\
& \cdot \exp \left\{\frac{\lambda}{2 \Lambda\left(A_{r}\right)}\left[b(T)-b\left(t_{n}\right)\right]\right. \\
& \left.\cdot\left\langle\vec{u}, A_{r}^{-1}\left\langle\vec{v} \alpha_{n+1}, \alpha_{n+1}\right\rangle\right\rangle_{\mathbb{R}}^{2}\right\} .
\end{aligned}
$$

The following lemmas are useful to prove the results in the next sections [5].

Lemma 1. Let $v \in L_{2}[0, T]$. Then for $w_{\varphi}$ a.e. $x \in C[0, T]$

$$
\begin{aligned}
(v, X(x, \cdot)) & =\left(M_{h} v, x\right), \\
\left(v, P_{b, n+1}(X(x, \cdot))\right) & =\left(\mathscr{P} M_{h} v, x\right),
\end{aligned}
$$

where $M_{h}: L_{2}[0, T] \rightarrow L_{2}[0, T]$ is the multiplication operator defined by

$$
M_{h} u=h u \quad \text { for } u \in L_{2}[0, T] \text {. }
$$

Lemma 2. Let $v \in L_{2}[0, T]$ and $\vec{\xi}_{n+1}=\left(\xi_{0}, \xi_{1}, \ldots, \xi_{n}, \xi_{n+1}\right) \in$ $\mathbb{R}^{n+2}$. Then

$$
\begin{aligned}
\left(v, P_{b, n+1}\left(\vec{\xi}_{n+1}\right)\right)= & \left(v, P_{b, n}\left(\vec{\xi}_{n}\right)\right) \\
& +\left\langle v \alpha_{n+1}, \alpha_{n+1}\right\rangle\left(\xi_{n+1}-\xi_{n}\right),
\end{aligned}
$$

where $\vec{\xi}_{n}=\left(\xi_{0}, \xi_{1}, \ldots, \xi_{n}\right)$.
Lemma 3. Let $\left\{v_{1}, \ldots, v_{r}\right\}$ be a subset of $L_{2}[0, T]$ such that $\left\{M_{h} v_{1}, \ldots, M_{h} v_{r}\right\}$ is an independent set. Then the random vector $(\vec{v}, Z(x, \cdot)) \equiv\left(\left(v_{1}, Z(x, \cdot)\right), \ldots,\left(v_{r}, Z(x, \cdot)\right)\right)$ has the multivariate normal distribution with mean vector $(\vec{v}, a) \equiv$ $\left(\left(v_{1}, a\right), \ldots,\left(v_{r}, a\right)\right)$ and covariance matrix $\Sigma_{M_{h}}=\left[\left\langle M_{h} v_{i}\right.\right.$, $\left.\left.M_{h} v_{j}\right\rangle\right]_{r \times r}$. Moreover, for any Borel measurable function $f$ : $\mathbb{R}^{r+1} \rightarrow \mathbb{C}$ one has

$$
\begin{aligned}
& \int_{C[0, T]} f(x(0),(\vec{v}, Z(x, \cdot))) d w_{\varphi}(x) \\
& \stackrel{*}{=} \int_{\mathbb{R}} \int_{\mathbb{R}^{r}} f\left(u_{0}, \vec{u}\right) \Psi_{r}\left(1,(\vec{v}, a), \Sigma_{M_{h}}, \vec{u}\right) d \vec{u} d \varphi\left(u_{0}\right),
\end{aligned}
$$

where $\stackrel{*}{=}$ means that if either side exists, then both sides exist and they are equal.

Remark 4. Because the Borel sets of $C[0, T]$ are always scaleinvariant measurable and we use the Borel class of $C[0, T]$ on which $w_{\varphi}$ is defined [1], the scale-invariant measurability is not essential in (7).

\section{Conditional Fourier-Wiener and Fourier- Feynman Transforms}

Let $1 \leq p \leq \infty$, let $r$ be any fixed positive integer, and let $\left\{v_{1}, v_{2}, \ldots, v_{r}\right\}$ be an orthonormal subset of $L_{2}[0, T]$ such that both $\left\{M_{h} v_{1}, \ldots, M_{h} v_{r}\right\}$ and $\left\{\mathscr{P}^{\perp} M_{h} v_{1}, \ldots, \mathscr{P}^{\perp} M_{h} v_{r}\right\}$ are independent sets. Let $\mathscr{A}^{(p)}$ be the space of cylinder functions $F$ having the form

$$
F(x)=f((\vec{v}, x))
$$

for $w_{\varphi}$ a.e. $x \in C[0, T]$, where $f \in L_{p}\left(\mathbb{R}^{r}\right)$ and $(\vec{v}, x)=\left(\left(v_{1}\right.\right.$, $\left.x), \ldots,\left(v_{r}, x\right)\right)$. Without loss of generality we can take $f$ to be Borel measurable.

Theorem 5. Let $1 \leq p \leq \infty$ and let $F\left(\in \mathscr{A}^{(p)}\right)$ be given by (29). Then for $\lambda \in \mathbb{C}_{+}$

$$
\begin{aligned}
T_{\lambda} & {\left[F_{Z} \mid Z_{n}\right]\left(y, \vec{\xi}_{n}\right) } \\
& =\int_{\mathbb{R}^{r}} f\left(\sum_{\mathscr{P} \perp}^{1 / 2} \vec{u}+\left(\vec{v}, y+a+P_{b, n}\left(\vec{\xi}_{n}-\vec{a}_{n}\right)\right)\right) \\
& \cdot \Psi_{r}\left(\lambda, \overrightarrow{0}, I_{r}, \vec{u}\right) \Phi_{r}\left(\lambda, \vec{v}, \Sigma_{\mathscr{P} \perp}^{1 / 2}, \vec{u}\right) d \vec{u} \\
& =\left(f_{r}\left(\vec{\xi}_{n}-\vec{a}_{n}, a, \cdot\right)\right. \\
& \left.*\left(\Psi_{r}\left(\lambda, \overrightarrow{0}, I_{r}, \cdot\right) \Phi_{r}\left(\lambda, \vec{v}, \Sigma_{\mathcal{P}^{\perp}}^{1 / 2}, \cdot\right)\right)\right)\left(\Sigma_{\mathscr{P}^{\perp}}^{-1 / 2}(\vec{v}, y)\right),
\end{aligned}
$$

for $w_{\varphi}$ a.e. $y \in C[0, T]$ and $P_{Z_{n}}$ a.e. $\vec{\xi}_{n} \in \mathbb{R}^{n+1}$, where $\Sigma_{\mathscr{P}^{\perp}}=$ $\left[\left\langle\mathscr{P}^{\perp} M_{h} v_{i}, \mathscr{P}^{\perp} M_{h} v_{j}\right\rangle\right], \vec{a}_{n}=\left(a\left(t_{0}\right), a\left(t_{1}\right), \ldots, a\left(t_{n}\right)\right)$, and

$$
f_{r}\left(\vec{\xi}_{n}, a, \vec{u}\right)=f\left(\sum_{\mathscr{P} \perp}^{1 / 2} \vec{u}+\left(\vec{v}, a+P_{b, n}\left(\vec{\xi}_{n}\right)\right)\right) .
$$

Moreover $T_{\lambda}\left[F_{Z} \mid Z_{n}\right]\left(\cdot, \vec{\xi}_{n}\right) \in \mathscr{A}^{(p)}$. 
Proof. For $\vec{\xi}_{n}=\left(\xi_{0}, \xi_{1}, \ldots, \xi_{n}\right) \in \mathbb{R}^{n+1}$ let $\vec{\xi}_{n+1}=\left(\xi_{0}, \xi_{1}, \ldots\right.$, $\left.\xi_{n}, \xi_{n+1}\right)$, where $\xi_{n+1} \in \mathbb{R}$. For $\lambda>0$ and $w_{\varphi}$ a.e. $x \in C[0, T]$ we have by Lemmas 1 and 2

$$
\begin{aligned}
& I_{F_{Z}}^{\lambda}\left(y, \vec{\xi}_{n}\right)=\int_{\mathbb{R}} \int_{\mathbb{R}^{r}} \Psi_{1}\left(\lambda, a(T)-a\left(t_{n}\right), b(T)\right. \\
& \left.\quad-b\left(t_{n}\right), \xi_{n+1}-\xi_{n}\right) f\left(\Sigma_{\mathscr{P} \boldsymbol{\lrcorner}}^{1 / 2} \vec{u}+(\vec{v}, y)\right. \\
& \left.\quad+\left(\vec{v}, A+P_{b, n+1}\left(\vec{\xi}_{n+1}\right)\right)\right) \\
& \quad+\Psi_{r}\left(\lambda, \overrightarrow{0}, I_{r}, \vec{u}\right) d \vec{u} d \xi_{n+1} \\
& \quad=\int_{\mathbb{R}^{r}} \int_{\mathbb{R}} \Psi_{1}\left(\lambda, 0, b(T)-b\left(t_{n}\right), \xi_{n+1}\right) f\left(\Sigma_{\mathscr{P} \perp}^{1 / 2} \vec{u}\right. \\
& \quad+(\vec{v}, y)+(\vec{v}, A)+\left(\vec{v}, P_{b, n}\left(\vec{\xi}_{n}\right)\right) \\
& \quad+\left(a(T)-a\left(t_{n}\right)\right)\left\langle\vec{v} \alpha_{n+1}, \alpha_{n+1}\right\rangle \\
& \left.\quad+\left\langle\vec{v} \alpha_{n+1}, \alpha_{n+1}\right\rangle \xi_{n+1}\right) \\
& \quad . \Psi_{r}\left(\lambda, \overrightarrow{0}, I_{r}, \vec{u}\right) d \xi_{n+1} d \vec{u},
\end{aligned}
$$

where $A$ and $\Psi_{r}$ are given by (12) and (13), respectively. Using the same method as used in the proof of Theorem 3.2 in [7]

$$
\begin{aligned}
& I_{F_{Z}}^{\lambda}\left(y, \vec{\xi}_{n}\right)=\int_{\mathbb{R}^{r}} f\left(\Sigma_{\mathscr{P} \perp}^{1 / 2} \vec{u}+(\vec{v}, y)+(\vec{v}, A)\right. \\
& \left.+\left(\vec{v}, P_{b, n}\left(\vec{\xi}_{n}\right)\right)+\left(a(T)-a\left(t_{n}\right)\right)\left\langle\vec{v} \alpha_{n+1}, \alpha_{n+1}\right\rangle\right) \\
& . \Psi_{r}\left(\lambda, \overrightarrow{0}, I_{r}, \vec{u}\right) \Phi_{r}\left(\lambda, \vec{v}, \Sigma_{\mathscr{P} \perp}^{1 / 2}, \vec{u}\right) d \vec{u} \\
& \quad=\int_{\mathbb{R}^{r}} f\left(\Sigma_{\mathscr{P}^{\perp}}^{1 / 2} \vec{u}+\left(\vec{v}, y+a+P_{b, n}\left(\vec{\xi}_{n}-\vec{a}_{n}\right)\right)\right) \\
& . \Psi_{r}\left(\lambda, \overrightarrow{0}, I_{r}, \vec{u}\right) \Phi_{r}\left(\lambda, \vec{v}, \Sigma_{\mathscr{P} \perp}^{1 / 2}, \vec{u}\right) d \vec{u} .
\end{aligned}
$$

We note that if $1 \leq p<\infty$, then by the change of variable theorem

$$
\left\|f_{r}\left(\vec{\xi}_{n}-\vec{a}_{n}, a, \cdot\right)\right\|_{p}^{p}=\left|\Sigma_{\mathscr{P} \perp}^{-1 / 2}\right|\|f\|_{p}^{p}<\infty,
$$

and for $\lambda \in \mathbb{C}_{+}$we have by Schwarz's inequality

$$
\begin{aligned}
& \left|\Psi_{r}\left(\lambda, \overrightarrow{0}, I_{r}, \vec{u}\right) \Phi_{r}\left(\lambda, \vec{v}, \Sigma_{\mathscr{P} \perp}^{1 / 2}, \vec{u}\right)\right| \\
& \quad \leq\left(\frac{|\lambda|}{2 \pi}\right)^{r / 2} \exp \left\{-\frac{\operatorname{Re} \lambda}{2 \Lambda\left(\Sigma_{\mathscr{P} \perp}^{1 / 2}\right)}\|\vec{u}\|_{\mathbb{R}}^{2}\right\} .
\end{aligned}
$$

Now, by Morera's theorem with aids of Hölder's inequality and the dominated convergence theorem, we have (30) for $\lambda \in \mathbb{C}_{+}$. Since $f_{r}\left(\vec{\xi}_{n}, a, \cdot\right) \in L_{p}\left(\mathbb{R}^{r}\right)$ and $\Psi_{r}(\lambda, \overrightarrow{0}$, $\left.I_{r}, \cdot\right) \Phi_{r}\left(\lambda, \vec{v}, \Sigma_{\mathscr{P} \perp}^{1 / 2}, \cdot\right) \in L_{1}\left(\mathbb{R}^{r}\right)$ by (35), the final result follows by the change of variable theorem and Young's inequality [9].

Corollary 6. Let $1 \leq p \leq \infty$ and let $F\left(\in \mathscr{A}^{(p)}\right)$ be given by (29).
(1) If $n=0$, then one has for $\lambda \in \mathbb{C}_{+}, y \in C[0, T]$ and $\xi_{0} \in \mathbb{R}$

$$
\begin{aligned}
& T_{\lambda}\left[F_{Z} \mid Z_{0}\right]\left(y, \xi_{0}\right)=\int_{\mathbb{R}^{r}} f\left(\Sigma_{\mathscr{P} \perp}^{1 / 2} \vec{u}+(\vec{v}, y+a)\right) \\
& \cdot \Psi_{r}\left(\lambda, \overrightarrow{0}, I_{r}, \vec{u}\right) \Phi_{r}^{0}\left(\lambda, \vec{v}, \Sigma_{\mathscr{P}^{\perp}}^{1 / 2} \vec{u}\right) d \vec{u}, \\
& \text { where for } \Lambda^{0}\left(\Sigma_{\mathscr{P}^{\perp}}^{1 / 2}\right)=1+b(T)\left\|\Sigma_{\mathscr{P} \perp}^{-1 / 2}\left\langle\vec{v} \alpha_{1}, \alpha_{1}\right\rangle\right\|_{\mathbb{R}}^{2} \\
& \Phi_{r}^{0}\left(\lambda, \vec{v}, \Sigma_{\mathscr{P}^{\perp}}^{1 / 2}, \vec{u}\right)=\left[\frac{1}{\Lambda^{0}\left(\Sigma_{\mathscr{P}^{\perp}}^{1 / 2}\right)}\right]^{1 / 2} \\
& \cdot \exp \left\{\frac{\lambda b(T)}{2 \Lambda^{0}\left(\Sigma_{\mathscr{P}^{\perp}}^{1 / 2}\right)}\left\langle\vec{u}, \Sigma_{\mathscr{P}^{\perp}}^{-1 / 2}\left\langle\vec{v} \alpha_{1}, \alpha_{1}\right\rangle\right\rangle_{\mathbb{R}}^{2}\right\} .
\end{aligned}
$$

(2) If $r=1$, then one has

$$
\begin{aligned}
T_{\lambda} & {\left[F_{Z} \mid Z_{n}\right]\left(y, \vec{\xi}_{n}\right)=\int_{\mathbb{R}} f\left(\left\|\mathscr{P}^{\perp} M_{h} v_{1}\right\| u\right.} \\
& \left.+\left(v_{1}, y+a+P_{b, n}\left(\vec{\xi}_{n}-\vec{a}_{n}\right)\right)\right) \Delta_{1}(\lambda, u) d u \\
& =\left(f_{1}\left(\vec{\xi}_{n}-\vec{a}_{n}, a, \cdot\right) * \Delta_{1}(\lambda, \cdot)\right)\left(\left\|\mathscr{P}^{\perp} M_{h} v_{1}\right\|^{-1}\right. \\
& \left.\cdot\left(v_{1}, y\right)\right)
\end{aligned}
$$

where for $u \in \mathbb{R}$

$$
\begin{aligned}
& f_{1}\left(\vec{\xi}_{n}, a, u\right) \\
& \quad=f\left(\left\|\mathscr{P}^{\perp} M_{h} v_{1}\right\| u+\left(v_{1}, a+P_{b, n}\left(\vec{\xi}_{n}\right)\right)\right),
\end{aligned}
$$

and for $\Lambda_{1}\left(\mathscr{P}^{\perp} M_{h}\right)=1+[b(T)-$ $\left.b\left(t_{n}\right)\right]\left(\left\|\mathscr{P}^{\perp} M_{h} v_{1}\right\|^{-1}\left\langle v_{1} \alpha_{n+1}, \alpha_{n+1}\right\rangle\right)^{2}$

$\Delta_{1}(\lambda, u)$

$$
=\left[\frac{\lambda}{2 \pi \Lambda_{1}\left(\mathscr{P}^{\perp} M_{h}\right)}\right]^{1 / 2} \exp \left\{-\frac{\lambda u^{2}}{2 \Lambda_{1}\left(\mathscr{P}^{\perp} M_{h}\right)}\right\} .
$$

(3) If $n=0$ and $r=1$, then one has

$$
\begin{aligned}
T_{\lambda} & {\left[F_{Z} \mid Z_{0}\right]\left(y, \xi_{0}\right) } \\
& =\int_{\mathbb{R}} f\left(\left\|\mathscr{P}^{\perp} M_{h} v_{1}\right\| u+\left(v_{1}, y+a\right)\right) \Delta_{1}^{0}(\lambda, u) d u \\
& =\left(f_{1}^{0}(a, \cdot) * \Delta_{1}^{0}(\lambda, \cdot)\right)\left(\left\|\mathscr{P}^{\perp} M_{h} v_{1}\right\|^{-1}\left(v_{1}, y\right)\right),
\end{aligned}
$$

where for $u \in \mathbb{R}$

$$
f_{1}^{0}(a, u)=f\left(\left\|\mathscr{P}^{\perp} M_{h} v_{1}\right\| u+\left(v_{1}, a\right)\right),
$$




$$
\begin{aligned}
& \quad \text { and for } \Lambda_{1}^{0}\left(\mathscr{P}^{\perp} M_{h}\right)=1+b(T)\left(\| \mathscr { P } ^ { \perp } M _ { h } v _ { 1 } \| ^ { - 1 } \left\langlev_{1} \alpha_{1},\right.\right. \\
& \left.\left.\quad \alpha_{1}\right\rangle\right)^{2} \\
& \Delta_{1}^{0}(\lambda, u) \\
& =\left[\frac{\lambda}{2 \pi \Lambda_{1}^{0}\left(\mathscr{P}^{\perp} M_{h}\right)}\right]^{1 / 2} \exp \left\{-\frac{\lambda u^{2}}{2 \Lambda_{1}^{0}\left(\mathscr{P}^{\perp} M_{h}\right)}\right\} .
\end{aligned}
$$

By the dominated convergence theorem and Theorem 5 we have the following theorem.

Theorem 7. Let $F\left(\in \mathscr{A}^{(1)}\right)$ be given by (29). Then for a nonzero real $q$, $w_{\varphi}$ a.e. $y \in C[0, T]$, and $P_{Z_{n}}$ a.e. $\vec{\xi}_{n} \in \mathbb{R}^{n+1}, T_{q}^{(1)}\left[F_{Z} \mid\right.$ $\left.Z_{n}\right]\left(y, \vec{\xi}_{n}\right)$ exists and it is given by the right-hand side of (30), where $\lambda$ is replaced by-iq. Furthermore $T_{q}^{(1)}\left[F_{Z} \mid Z_{n}\right]\left(\cdot, \vec{\xi}_{n}\right) \in$ $\mathscr{A}^{(\infty)}$.

Using Lemma 1.2 in [10] we can prove the following theorem.

Theorem 8. Let $F\left(\in \mathscr{A}^{(p)}\right)$ be given by (29) with $1<p \leq 2$, let $q$ be a nonzero real number, and let $1 / p+1 / p^{\prime}=1$.

(1) Suppose that $\left\langle v_{j} \alpha_{n+1}, \alpha_{n+1}\right\rangle=0$ for $j=1, \ldots, r$. Then for $w_{\varphi}$ a.e. $y \in C[0, T]$ and $P_{Z_{n}}$ a.e. $\vec{\xi}_{n} \in \mathbb{R}^{n+1}$, $T_{q}^{(p)}\left[F_{Z} \mid Z_{n}\right]\left(y, \vec{\xi}_{n}\right)$ is given by

$$
\begin{aligned}
T_{q}^{(p)} & {\left[F_{Z} \mid Z_{n}\right]\left(y, \vec{\xi}_{n}\right) } \\
& =\int_{\mathbb{R}^{r}} f\left(\Sigma_{\mathscr{P} \perp}^{1 / 2} \vec{u}+\left(\vec{v}, y+a+P_{b, n}\left(\vec{\xi}_{n}-\vec{a}_{n}\right)\right)\right) \\
& \cdot \Psi_{r}\left(\lambda, \overrightarrow{0}, I_{r}, \vec{u}\right) d \vec{u}=\left(f_{r}\left(\vec{\xi}_{n}-\vec{a}_{n}, a, \cdot\right)\right. \\
& \left.* \Psi_{r}\left(-i q, \overrightarrow{0}, I_{r}, \cdot\right)\right)\left(\Sigma_{\mathscr{P} \perp}^{-1 / 2}(\vec{v}, y)\right),
\end{aligned}
$$

where $f_{r}$ is given by (31). Furthermore $T_{q}^{(p)}\left[F_{Z} \mid Z_{n}\right](\cdot$, $\left.\vec{\xi}_{n}\right) \in \mathscr{A}^{\left(p^{\prime}\right)}$.

(2) If $r=1$, then for $w_{\varphi}$ a.e. $y \in C[0, T]$ and $P_{Z_{n}}$ a.e. $\vec{\xi}_{n} \in \mathbb{R}^{n+1}, T_{q}^{(p)}\left[F_{Z} \mid Z_{n}\right]\left(y, \vec{\xi}_{n}\right)$ exists and it is given by the right-hand sides of (38), where $\lambda$ is replaced by-iq. Furthermore $T_{q}^{(p)}\left[F_{Z} \mid Z_{n}\right]\left(\cdot, \vec{\xi}_{n}\right) \in \mathscr{A}^{\left(p^{\prime}\right)}$.

(3) If $n=0$ and $r=1$, then for $\xi_{0} \in \mathbb{R}, T_{q}^{(p)}\left[F_{Z} \mid Z_{0}\right](y$, $\left.\xi_{0}\right)$ is given by the right-hand sides of (41), where $\lambda$ is replaced by $-i q$.

Using the same method as used in the proof of Theorem 4.2 in [7], we can prove the following theorem.

Theorem 9. Let $F\left(\in \mathscr{A}^{(p)}\right)$ be given by (29) with $1 \leq p \leq \infty$, and let $q \in \mathbb{R}-\{0\}$.

(1) Suppose that $\left\langle v_{j} \alpha_{n+1}, \alpha_{n+1}\right\rangle=0$ for $j=1, \ldots, r$. For $w_{\varphi}$ a.e. $y \in C[0, T]$ and $P_{Z_{n}}$ a.e. $\vec{\xi}_{n}, \vec{\eta}_{n} \in \mathbb{R}^{n+1}$, let
$F_{r}\left(y, \vec{\xi}_{n}, \vec{\eta}_{n}\right)=f\left((\vec{v}, y)+\left(\vec{v}, 2 a+P_{b, n}\left(\vec{\xi}_{n}+\vec{\eta}_{n}-2 \vec{a}_{n}\right)\right)\right)$. Then

$$
\begin{gathered}
\int_{C[0, T]} \mid T_{\bar{\lambda}}\left[T_{\lambda}\left[F_{Z} \mid Z_{n}\right]\left(\cdot, \vec{\xi}_{n}\right) \mid Z_{n}\right]\left(y, \vec{\eta}_{n}\right) \\
-\left.F_{r}\left(y, \vec{\xi}_{n}, \vec{\eta}_{n}\right)\right|^{p} d w_{\varphi}(y) \longrightarrow 0,
\end{gathered}
$$

for $1 \leq p<\infty$, and for $1 \leq p \leq \infty$

$$
\begin{aligned}
T_{\bar{\lambda}} & {\left[T_{\lambda}\left[F_{Z} \mid Z_{n}\right]\left(\cdot, \vec{\xi}_{n}\right) \mid Z_{n}\right]\left(y, \vec{\eta}_{n}\right) } \\
& \longrightarrow F_{r}\left(y, \vec{\xi}_{n}, \vec{\eta}_{n}\right)
\end{aligned}
$$

as $\lambda$ approaches -iq through $\mathbb{C}_{+}$.

(2) Suppose that $r=1$. Let $F_{1}\left(y, \vec{\xi}_{n}, \vec{\eta}_{n}\right)=f\left(\left(v_{1}, y\right)+\left(v_{1}\right.\right.$, $\left.\left.2 a+P_{b, n}\left(\vec{\xi}_{n}+\vec{\eta}_{n}-2 \vec{a}_{n}\right)\right)\right)$. Then one has (45) if $1 \leq p<\infty$ and has (46) if $1 \leq p \leq \infty$, where $F_{r}$ is replaced by $F_{1}$.

(3) Suppose that $n=0$ and $r=1$. Let $F_{1}^{0}\left(y, \xi_{0}, \eta_{0}\right)=$ $f\left(\left(v_{1}, y\right)+\left(v_{1}, 2 a\right)\right)$. Then one has (45) if $1 \leq p<\infty$ and has (46) if $1 \leq p \leq \infty$, where $F_{r}$ is replaced by $F_{1}^{0}$.

\section{Relationships between Conditional Fourier-Feynman Transforms and Convolution Products}

In this section we evaluate the conditional convolution products and investigate their relationships.

Now, we have the following two theorems by (35) and Theorems 3.2 and 3.3 in [7].

Theorem 10. Let $F\left(\in \mathscr{A}^{\left(p_{1}\right)}\right), G\left(\in \mathscr{A}^{\left(p_{2}\right)}\right)$, and $f$ and $g$ be related by (29), respectively, where $1 \leq p_{1}, p_{2} \leq \infty$. Furthermore let $1 / p_{1}+1 / p_{1}^{\prime}=1$ and $1 / p_{2}+1 / p_{2}^{\prime}=1$. Then for $\lambda \in \mathbb{C}_{+}$, $w_{\varphi}$ a.e. $y \in C[0, T]$, and $P_{Z_{n}}$ a.e. $\vec{\xi}_{n} \in \mathbb{R}^{n+1},\left[\left(F_{Z} * G_{Z}\right)_{\lambda} \mid\right.$ $\left.Z_{n}\right]\left(y, \vec{\xi}_{n}\right)$ exists and is given by

$$
\begin{aligned}
& {\left[\left(F_{Z} * G_{Z}\right)_{\lambda} \mid Z_{n}\right]\left(y, \vec{\xi}_{n}\right)=\int_{\mathbb{R}^{r}} f\left(\frac{1}{\sqrt{2}}[(\vec{v}, y)\right.} \\
& \left.\left.\quad+\sum_{\mathscr{P}^{\perp}}^{1 / 2} \vec{u}+\left(\vec{v}, a+P_{b, n}\left(\vec{\xi}_{n}-\vec{a}_{n}\right)\right)\right]\right) g\left(\frac{1}{\sqrt{2}}[(\vec{v}, y)\right. \\
& \left.\left.\quad-\sum_{\mathscr{P} \perp}^{1 / 2} \vec{u}-\left(\vec{v}, a+P_{b, n}\left(\vec{\xi}_{n}-\vec{a}_{n}\right)\right)\right]\right) \Psi_{r}\left(\lambda, \overrightarrow{0}, I_{r}, \vec{u}\right) \\
& \quad . \Phi_{r}\left(\lambda, \vec{v}, \Sigma_{\mathscr{P} \perp}^{1 / 2}, \vec{u}\right) d \vec{u} .
\end{aligned}
$$

Moreover one has $\left[\left(F_{Z} * G_{Z}\right)_{\lambda} \mid Z_{n}\right]\left(\cdot, \vec{\xi}_{n}\right) \in \mathscr{A}^{(1)}$ if either $p_{2} \leq p_{1}^{\prime}$ or $p_{1} \leq p_{2}^{\prime},\left[\left(F_{Z} * G_{Z}\right)_{\lambda} \mid Z_{n}\right]\left(\cdot, \vec{\xi}_{n}\right) \in \mathscr{A}^{\left(p_{2}\right)}$ if $p_{2} \geq p_{1}^{\prime}$, and $\left[\left(F_{Z} * G_{Z}\right)_{\lambda} \mid Z_{n}\right]\left(\cdot, \vec{\xi}_{n}\right) \in \mathscr{A}^{\left(p_{1}\right)}$ if $p_{1} \geq p_{2}^{\prime}$. 
Theorem 11. Let $q$ be a nonzero real number. Then for $\lambda \in \mathbb{C}_{+}$ or $\lambda=q$ and $P_{Z_{n}}$ a.e. $\vec{\xi}_{n} \in \mathbb{R}^{n+1}$, one has the following:

(1) if $F, G \in \mathscr{A}^{(1)}$, then $\left[\left(F_{Z} * G_{Z}\right)_{\lambda} \mid Z_{n}\right]\left(\cdot, \vec{\xi}_{n}\right) \in \mathscr{A}^{(1)}$,

(2) if $F, G \in \mathscr{A}^{(2)}$, then $\left[\left(F_{Z} * G_{Z}\right)_{\lambda} \mid Z_{n}\right]\left(\cdot, \vec{\xi}_{n}\right) \in \mathscr{A}^{(\infty)}$,

(3) if $F \in \mathscr{A}^{(1)}$ and $G \in \mathscr{A}^{(2)}$, then $\left[\left(F_{Z} * G_{Z}\right)_{\lambda} \mid Z_{n}\right](\cdot$, $\left.\vec{\xi}_{n}\right) \in \mathscr{A}^{(2)}$

(4) if $F \in \mathscr{A}^{(1)}$ and $G \in \mathscr{A}^{(1)} \cap \mathscr{A}^{(2)}$, then $\left[\left(F_{Z} * G_{Z}\right)_{\lambda}\right.$ | $\left.Z_{n}\right]\left(\cdot, \vec{\xi}_{n}\right) \in \mathscr{A}^{(1)} \cap \mathscr{A}^{(2)}$,

(5) if $F \in \mathscr{A}^{(1)}$ and $G \in \mathscr{A}^{(\infty)}$, then $\left[\left(F_{Z} * G_{Z}\right)_{\lambda} \mid Z_{n}\right](\cdot$, $\left.\vec{\xi}_{n}\right) \in \mathscr{A}^{(\infty)}$.
Theorem 12. Let $F, G \in \bigcup_{1 \leq p \leq \infty} \mathscr{A}^{(p)}$ and let $\vec{a}_{n}=\left(a\left(t_{0}\right)\right.$, $\left.a\left(t_{1}\right), \ldots, a\left(t_{n}\right)\right)$. Then for $\lambda \in \mathbb{C}_{+}, w_{\varphi}$ a.e. $y \in C[0, T]$, and $P_{Z_{n}}$ a.e. $\vec{\xi}_{n}, \vec{\eta}_{n} \in \mathbb{R}^{n+1}$, one has

$$
\begin{aligned}
T_{\lambda} & {\left[\left[\left(F_{Z} * G_{Z}\right)_{\lambda} \mid Z_{n}\right]\left(\cdot, \vec{\xi}_{n}\right) \mid Z_{n}\right]\left(y, \vec{\eta}_{n}\right) } \\
& =\left[T _ { \lambda } [ F _ { Z } | Z _ { n } ] \left(\frac{1}{\sqrt{2}} y\right.\right. \\
& \left.\left.+(\sqrt{2}-1) a, \frac{1}{\sqrt{2}}\left(\vec{\eta}_{n}+\vec{\xi}_{n}\right)-(\sqrt{2}-1) \vec{a}_{n}\right)\right] \\
& \cdot\left[T_{\lambda}\left[G_{Z} \mid Z_{n}\right]\left(\frac{1}{\sqrt{2}} y-a, \frac{1}{\sqrt{2}}\left(\vec{\eta}_{n}-\vec{\xi}_{n}\right)+\vec{a}_{n}\right)\right] .
\end{aligned}
$$

Proof. We note that $T_{\lambda}\left[\left[\left(F_{Z} * G_{Z}\right)_{\lambda} \mid Z_{n}\right]\left(\cdot, \vec{\xi}_{n}\right) \mid Z_{n}\right]\left(y, \vec{\eta}_{n}\right)$ is well-defined by Theorems 5 and 10 . By those theorems as stated above we have for $\lambda \in \mathbb{C}_{+}, w_{\varphi}$ a.e. $y \in C[0, T]$, and $P_{Z_{n}}$ a.e. $\vec{\xi}_{n}, \vec{\eta}_{n} \in \mathbb{R}^{n+1}$

$$
\begin{aligned}
T_{\lambda} & {\left.\left[\left(F_{Z} * G_{Z}\right)_{\lambda} \mid Z_{n}\right]\left(\cdot, \vec{\xi}_{n}\right) \mid Z_{n}\right]\left(y, \vec{\eta}_{n}\right)=\int_{\mathbb{R}^{r}} \int_{\mathbb{R}^{r}} f\left(\frac{1}{\sqrt{2}}\left[(\vec{v}, y)+\Sigma_{\mathscr{P}^{\perp}}^{1 / 2}(\vec{u}+\vec{z})+\left(\vec{v}, 2 a+P_{b, n}\left(\vec{\xi}_{n}+\vec{\eta}_{n}-2 \vec{a}_{n}\right)\right)\right]\right) } \\
\cdot & g\left(\frac{1}{\sqrt{2}}\left[(\vec{v}, y)+\Sigma_{\mathscr{P}^{\perp}}^{1 / 2}(\vec{z}-\vec{u})+\left(\vec{v}, P_{b, n}\left(\vec{\eta}_{n}-\vec{\xi}_{n}\right)\right)\right]\right) \Psi_{r}\left(\lambda, \overrightarrow{0}, I_{r}, \vec{u}\right) \Phi_{r}\left(\lambda, \vec{v}, \Sigma_{\mathscr{P} \perp}^{1 / 2} \vec{u}\right) \Psi_{r}\left(\lambda, \overrightarrow{0}, I_{r}, \vec{z}\right) \\
\cdot & \Phi_{r}\left(\lambda, \vec{v}, \Sigma_{\mathscr{P} \perp}^{1 / 2}, \vec{z}\right) d \vec{u} d \vec{z} .
\end{aligned}
$$

Let $\vec{\alpha}=(1 / \sqrt{2})(\vec{z}+\vec{u})$ and $\vec{\beta}=(1 / \sqrt{2})(\vec{z}-\vec{u})$. Then we have by the change of variable theorem and Lemma 2

$$
\begin{aligned}
& T_{\lambda}\left[\left[\left(F_{Z} * G_{Z}\right)_{\lambda} \mid Z_{n}\right]\left(\cdot, \vec{\xi}_{n}\right) \mid Z_{n}\right]\left(y, \vec{\eta}_{n}\right)=\int_{\mathbb{R}^{r}} \int_{\mathbb{R}^{r}} f\left(\Sigma_{\mathscr{P}^{\perp}}^{1 / 2} \vec{\alpha}+\frac{1}{\sqrt{2}}\left[\left(\vec{v}, y+2 a+P_{b, n}\left(\vec{\xi}_{n}+\vec{\eta}_{n}-2 \vec{a}_{n}\right)\right)\right]\right) g\left(\Sigma_{\mathscr{P}^{\perp}}^{1 / 2} \vec{\beta}\right. \\
& \left.+\frac{1}{\sqrt{2}}\left[\left(\vec{v}, y+P_{b, n}\left(\vec{\eta}_{n}-\vec{\xi}_{n}\right)\right)\right]\right) \Psi_{r}\left(\lambda, \overrightarrow{0}, I_{r}, \frac{1}{\sqrt{2}}(\vec{\alpha}+\vec{\beta})\right) \Phi_{r}\left(\lambda, \vec{v}, \Sigma_{\mathscr{P}^{\perp}}^{1 / 2}, \frac{1}{\sqrt{2}}(\vec{\alpha}+\vec{\beta})\right) \Psi_{r}\left(\lambda, \overrightarrow{0}, I_{r}, \frac{1}{\sqrt{2}}(\vec{\alpha}-\vec{\beta})\right) \\
& \cdot \Phi_{r}\left(\lambda, \vec{v}, \Sigma_{\mathscr{P}^{\perp}}^{1 / 2}, \frac{1}{\sqrt{2}}(\vec{\alpha}-\vec{\beta})\right) d \vec{\alpha} d \vec{\beta}=\frac{\lambda^{r}}{(2 \pi)^{r} \Lambda\left(\Sigma_{\mathscr{P}^{\perp}}^{1 / 2}\right)} \int_{\mathbb{R}^{r}} \int_{\mathbb{R}^{r}} f\left(\Sigma_{\mathscr{P}^{\perp}}^{1 / 2} \vec{\alpha}+\left(\vec{v}, \frac{1}{\sqrt{2}} y+\sqrt{2} a+P_{b, n}\left(\frac{1}{\sqrt{2}}\left(\vec{\xi}_{n}+\vec{\eta}_{n}\right)-\sqrt{2} \vec{a}_{n}\right)\right)\right) \\
& \cdot g\left(\Sigma_{\mathscr{P}^{\perp}}^{1 / 2} \vec{\beta}+\left(\vec{v}, \frac{1}{\sqrt{2}} y+P_{b, n}\left(\frac{1}{\sqrt{2}}\left(\vec{\xi}_{n}-\vec{\eta}_{n}\right)\right)\right)\right) \\
& \cdot \exp \left\{-\frac{\lambda}{4}\left[\|\vec{\alpha}+\vec{\beta}\|_{\mathbb{R}}^{2}+\|\vec{\alpha}-\vec{\beta}\|_{\mathbb{R}}^{2}\right]+\frac{\lambda}{4 \Lambda\left(\Sigma_{\mathscr{P}^{\perp}}^{1 / 2}\right)}\left[b(T)-b\left(t_{n}\right)\right]\left[\left\langle\vec{\alpha}+\vec{\beta}, \Sigma_{\mathscr{\rho}^{\perp}}^{-1 / 2}\left\langle\vec{v} \alpha_{n+1}, \alpha_{n+1}\right\rangle\right\rangle_{\mathbb{R}}^{2}+\left\langle\vec{\alpha}-\vec{\beta}, \Sigma_{\mathscr{P}^{\perp}}^{-1 / 2}\left\langle\vec{\gamma} \alpha_{n+1}, \alpha_{n+1}\right\rangle\right\rangle_{\mathbb{R}}^{2}\right]\right\} d \vec{\alpha} d \vec{\beta} \\
& =\left[T_{\lambda}\left[F_{Z} \mid Z_{n}\right]\left(\frac{1}{\sqrt{2}} y+(\sqrt{2}-1) a, \frac{1}{\sqrt{2}}\left(\vec{\eta}_{n}+\vec{\xi}_{n}\right)-(\sqrt{2}-1) \vec{a}_{n}\right)\right]\left[T_{\lambda}\left[G_{Z} \mid Z_{n}\right]\left(\frac{1}{\sqrt{2}} y-a, \frac{1}{\sqrt{2}}\left(\vec{\eta}_{n}-\vec{\xi}_{n}\right)+\vec{a}_{n}\right)\right],
\end{aligned}
$$

which completes the proof.

We now have the following theorem from Theorems 5, 10, 11 , and 12 .

Theorem 13. Let q be a nonzero real number. Then one has the following:
(1) if $F, G \in \mathscr{A}^{(1)}$, then one has for $w_{\varphi}$ a.e. $y \in C[0, T]$ and $P_{Z_{n}}$ a.e. $\vec{\xi}_{n}, \vec{\eta}_{n} \in \mathbb{R}^{n+1}$

$$
\begin{aligned}
T_{q}^{(1)} & {\left[\left[\left(F_{Z} * G_{Z}\right)_{q} \mid Z_{n}\right]\left(\cdot, \vec{\xi}_{n}\right) \mid Z_{n}\right]\left(y, \vec{\eta}_{n}\right) } \\
& =\left[T _ { q } ^ { ( 1 ) } [ F _ { Z } | Z _ { n } ] \left(\frac{1}{\sqrt{2}} y\right.\right.
\end{aligned}
$$




$$
\begin{aligned}
& \left.\left.+(\sqrt{2}-1) a, \frac{1}{\sqrt{2}}\left(\vec{\eta}_{n}+\vec{\xi}_{n}\right)-(\sqrt{2}-1) \vec{a}_{n}\right)\right] \\
& \cdot\left[T_{q}^{(1)}\left[G_{Z} \mid Z_{n}\right]\left(\frac{1}{\sqrt{2}} y-a, \frac{1}{\sqrt{2}}\left(\vec{\eta}_{n}-\vec{\xi}_{n}\right)+\vec{a}_{n}\right)\right],
\end{aligned}
$$

(2) if $F \in \mathscr{A}^{(1)}$ and $G \in \mathscr{A}^{(2)}$, then

$$
\begin{aligned}
T_{q}^{(2)} & {\left[\left[\left(F_{Z} * G_{Z}\right)_{q} \mid Z_{n}\right]\left(\cdot, \vec{\xi}_{n}\right) \mid Z_{n}\right]\left(y, \vec{\eta}_{n}\right) } \\
& =\left[T _ { q } ^ { ( 1 ) } [ F _ { Z } | Z _ { n } ] \left(\frac{1}{\sqrt{2}} y\right.\right. \\
& \left.\left.+(\sqrt{2}-1) a, \frac{1}{\sqrt{2}}\left(\vec{\eta}_{n}+\vec{\xi}_{n}\right)-(\sqrt{2}-1) \vec{a}_{n}\right)\right] \\
& \cdot\left[T_{q}^{(2)}\left[G_{Z} \mid Z_{n}\right]\left(\frac{1}{\sqrt{2}} y-a, \frac{1}{\sqrt{2}}\left(\vec{\eta}_{n}-\vec{\xi}_{n}\right)+\vec{a}_{n}\right)\right] .
\end{aligned}
$$

$$
\begin{aligned}
T_{\lambda} & {\left[\Pi_{Z} \mid Z_{n}\right]\left(y, \vec{\xi}_{n}\right) } \\
& =\int_{\mathbb{R}^{r}} \exp \left\{-\frac{1}{2 \lambda}\left[\left[b(T)-b\left(t_{n}\right)\right]\left\langle\vec{z},\left\langle\vec{v} \alpha_{n+1}, \alpha_{n+1}\right\rangle\right\rangle_{\mathbb{R}}^{2}+\left\langle\sum_{\mathscr{P} \perp} \vec{z}, \vec{z}\right\rangle_{\mathbb{R}}\right]+i\left\langle\vec{z},\left(\vec{v}, y+a+P_{b, n}\left(\vec{\xi}_{n}-\vec{a}_{n}\right)\right)\right\rangle_{\mathbb{R}}\right\} d \rho(\vec{z}) .
\end{aligned}
$$

\section{Evaluation Formulas for the Functions in a Banach Algebra}

Let $\psi$ be the function defined by

$$
\psi(\vec{u})=\int_{\mathbb{R}^{r}} \exp \left\{i\langle\vec{u}, \vec{z}\rangle_{\mathbb{R}}\right\} d \rho(\vec{z}) \quad \text { for } \vec{u} \in \mathbb{R}^{r},
$$

where $\rho$ is a complex-valued Borel measure of bounded variation over $\mathbb{R}^{r}$. For $w_{\varphi}$ a.e. $x \in C[0, T]$, let $\Pi$ be given by

$$
\Pi(x)=\psi((\vec{v}, x)) .
$$

By Theorem 4.1 in [11], we can prove the following theorem.

Theorem 14. Let $1 \leq p \leq \infty$ and let $\Pi$ be given by (54). Then for $\lambda \in \mathbb{C}_{+}$, $w_{\varphi}$ a.e. $y \in C[0, T]$, and $P_{Z_{n}}$ a.e. $\vec{\xi}_{n} \in \mathbb{R}^{n+1}$, $T_{\lambda}\left[\Pi_{Z} \mid Z_{n}\right]\left(y, \vec{\xi}_{n}\right)$ is given by
For a nonzero real $q, T_{q}^{(p)}\left[\Pi_{Z} \mid Z_{n}\right]\left(y, \vec{\xi}_{n}\right)$ exists and it is given by the right-hand side of (55), where $\lambda$ is replaced by-iq. Furthermore $T_{\lambda}\left[\Pi_{Z} \mid Z_{n}\right]\left(\cdot, \vec{\xi}_{n}\right) \in \mathscr{A}^{(\infty)}$ and $T_{q}^{(p)}\left[\Pi_{Z} \mid Z_{n}\right](\cdot$, $\left.\vec{\xi}_{n}\right) \in \mathscr{A}^{(\infty)}$.

Theorem 15. Let $1 \leq p \leq \infty$ and let $\Pi$ be given by (54). Then one has that for $P_{Z_{n}}$ a.e. $\vec{\xi}_{n}, \vec{\eta}_{n} \in \mathbb{R}^{n+1}$

$$
\begin{aligned}
& \| T_{\bar{\lambda}}\left[T_{\lambda}\left[\Pi_{Z} \mid Z_{n}\right]\left(\cdot, \vec{\xi}_{n}\right) \mid Z_{n}\right]\left(\cdot, \vec{\eta}_{n}\right) \\
& \quad-\Pi\left(\cdot+2 a+P_{b, n}\left(\vec{\xi}_{n}+\vec{\eta}_{n}-2 \vec{a}_{n}\right)\right) \|_{p}
\end{aligned}
$$

converges to 0 and for $w_{\varphi}$ a.e. $y \in C[0, T]$

$$
\begin{aligned}
& T_{\bar{\lambda}}\left[T_{\lambda}\left[\Pi_{Z} \mid Z_{n}\right]\left(\cdot, \vec{\xi}_{n}\right) \mid Z_{n}\right]\left(y, \vec{\eta}_{n}\right) \\
& \longrightarrow \Pi\left(y+2 a+P_{b, n}\left(\vec{\xi}_{n}+\vec{\eta}_{n}-2 \vec{a}_{n}\right)\right),
\end{aligned}
$$

as $\lambda$ approaches -iq through $\mathbb{C}_{+}$.

Theorem 16. Let $\psi_{1}$ and $\psi_{2}$ and $\rho_{1}$ and $\rho_{2}$ be related by (53), respectively. Let $\Pi_{1}(x)=\psi_{1}((\vec{v}, x))$ and $\Pi_{2}(x)=\psi_{2}((\vec{v}, x))$ for $w_{\varphi}$ a.e. $x \in C[0, T]$. Then for $\lambda \in \mathbb{C}_{+}, w_{\varphi}$ a.e. $y \in C[0, T]$, and
$P_{Z_{n}}$ a.e. $\vec{\xi}_{n} \in \mathbb{R}^{n+1},\left[\left(\left(\Pi_{1}\right)_{Z} *\left(\Pi_{2}\right)_{Z}\right)_{\lambda} \mid Z_{n}\right]\left(y, \vec{\xi}_{n}\right)$ exists and it is given by

$$
\begin{aligned}
& {\left[\left(\left(\Pi_{1}\right)_{Z} *\left(\Pi_{2}\right)_{Z}\right)_{\lambda} \mid Z_{n}\right]\left(y, \vec{\xi}_{n}\right)} \\
& \quad=\int_{\mathbb{R}^{r}} \int_{\mathbb{R}^{r}} \exp \left\{-\frac{1}{4 \lambda}\left[\left[b(T)-b\left(t_{n}\right)\right]\right.\right. \\
& \quad \cdot\left\langle\left\langle\vec{v} \alpha_{n+1}, \alpha_{n+1}\right\rangle, \vec{u}-\vec{w}\right\rangle_{\mathbb{R}}^{2} \\
& \left.\quad+\left\|\Sigma_{\mathscr{P} \perp}^{1 / 2}(\vec{u}-\vec{w})\right\|_{\mathbb{R}}^{2}\right]+\frac{i}{\sqrt{2}}\left[\langle(\vec{v}, y), \vec{u}+\vec{w}\rangle_{\mathbb{R}}\right. \\
& \left.\left.\quad+\left\langle\left(\vec{v}, a+P_{b, n}\left(\vec{\xi}_{n}-\vec{a}_{n}\right)\right), \vec{u}-\vec{w}\right\rangle_{\mathbb{R}}\right]\right\} d \rho_{1}(\vec{u}) d \rho_{2}(\vec{w}) .
\end{aligned}
$$

For a nonzero real $q,\left[\left(\left(\Pi_{1}\right)_{Z} *\left(\Pi_{2}\right)_{Z}\right)_{q} \mid Z_{n}\right]\left(y, \vec{\xi}_{n}\right)$ is given by the above equation, where $\lambda$ is replaced by-iq. Furthermore, $\left[\left(\left(\Pi_{1}\right)_{Z} *\left(\Pi_{2}\right)_{Z}\right)_{q} \mid Z_{n}\right]\left(\cdot, \vec{\xi}_{n}\right) \in \mathscr{A}^{(\infty)}$.

Theorem 17. Let $q$ be a nonzero real number and let $1 \leq p \leq$ $\infty$. Furthermore let $\Pi_{1}$ and $\Pi_{2}$ be as given in Theorem 16. Then one has for $w_{\varphi}$ a.e. $y \in C[0, T]$ and $P_{Z_{n}}$ a.e. $\vec{\xi}_{n}, \vec{\eta}_{n} \in \mathbb{R}^{n+1}$

$$
\begin{aligned}
T_{q}^{(p)} & {\left[\left[\left(\left(\Pi_{1}\right)_{Z} *\left(\Pi_{2}\right)_{Z}\right)_{q} \mid Z_{n}\right]\left(\cdot, \vec{\xi}_{n}\right) \mid Z_{n}\right]\left(y, \vec{\eta}_{n}\right) } \\
& =\left[T _ { q } ^ { ( p ) } [ ( \Pi _ { 1 } ) _ { Z } | Z _ { n } ] \left(\frac{1}{\sqrt{2}} y\right.\right.
\end{aligned}
$$




$$
\begin{aligned}
& \left.\left.+(\sqrt{2}-1) a, \frac{1}{\sqrt{2}}\left(\vec{\eta}_{n}+\vec{\xi}_{n}\right)-(\sqrt{2}-1) \vec{a}_{n}\right)\right] \\
& \cdot\left[T _ { q } ^ { ( p ) } [ ( \Pi _ { 2 } ) _ { Z } | Z _ { n } ] \left(\frac{1}{\sqrt{2}} y-a, \frac{1}{\sqrt{2}}\left(\vec{\eta}_{n}-\vec{\xi}_{n}\right)\right.\right. \\
& \left.\left.+\vec{a}_{n}\right)\right] .
\end{aligned}
$$

Let $\mathscr{M}\left(L_{2}[0, T]\right)$ be the class of all complex-valued Borel measures of bounded variation over $L_{2}[0, T]$ and let $\mathcal{S}_{w_{\varphi}}$ be the space of all functions $F$ which for $\sigma \in \mathscr{M}\left(L_{2}[0, T]\right)$ have the form

$$
F(x)=\int_{L_{2}[0, T]} \exp \{i(v, x)\} d \sigma(v),
$$

for $w_{\varphi}$ a.e. $x \in C[0, T]$. Note that $\mathcal{S}_{w_{\varphi}}$ is a Banach algebra [1].

Theorem 18. Let $1 \leq p \leq \infty$ and let $F\left(\in \mathcal{S}_{w_{\varphi}}\right)$ be given by (60). Then for $\lambda \in \mathbb{C}_{+}, w_{\varphi}$ a.e. $y \in C[0, T]$, and $P_{Z_{n}}$ a.e. $\vec{\xi}_{n} \in$ $\mathbb{R}^{n+1}, T_{\lambda}\left[F_{Z} \mid Z_{n}\right]\left(y, \vec{\xi}_{n}\right)$ is given by

$$
\begin{aligned}
T_{\lambda} & {\left[F_{Z} \mid Z_{n}\right]\left(y, \vec{\xi}_{n}\right) } \\
& =\int_{L_{2}[0, T]} \exp \left\{-\frac{1}{2 \lambda}\left[\left[b(T)-b\left(t_{n}\right)\right]\left\langle v \alpha_{n+1}, \alpha_{n+1}\right\rangle^{2}+\left\|\mathscr{P}^{\perp} M_{h} v\right\|^{2}\right]+i\left(v, y+a+P_{b, n}\left(\vec{\xi}_{n}-\vec{a}_{n}\right)\right)\right\} d \sigma(v) .
\end{aligned}
$$

For a nonzero real $q, T_{q}^{(p)}\left[F_{Z} \mid Z_{n}\right]\left(y, \vec{\xi}_{n}\right)$ is given by the righthand side of the above equality, where $\lambda$ is replaced by-iq. Furthermore, $T_{q}^{(p)}\left[F_{Z} \mid Z_{n}\right]\left(\cdot, \vec{\xi}_{n}\right) \in \mathscr{A}^{(\infty)}$.
Proof. For $\vec{\xi}_{n}=\left(\xi_{0}, \xi_{1}, \ldots, \xi_{n}\right) \in \mathbb{R}^{n+1}$ let $\vec{\xi}_{n+1}=\left(\xi_{0}, \xi_{1}, \ldots\right.$, $\left.\xi_{n}, \xi_{n+1}\right)$, where $\xi_{n+1} \in \mathbb{R}$. Then for $\lambda>0, w_{\varphi}$ a.e. $y \in C[0, T]$, and $P_{Z_{n}}$ a.e. $\vec{\xi}_{n} \in \mathbb{R}^{n+1}$, we have by Lemmas 1 and 2

$$
\begin{aligned}
& I_{F_{Z}}^{\lambda}\left(y, \vec{\xi}_{n}\right)=\int_{\mathbb{R}} \Psi_{1}\left(\lambda, a(T)-a\left(t_{n}\right), b(T)-b\left(t_{n}\right), \xi_{n+1}-\xi_{n}\right) E\left[F\left(y+\lambda^{-1 / 2} X_{b, n+1}(x, \cdot)+A+P_{b, n+1}\left(\vec{\xi}_{n+1}\right)\right)\right] d \xi_{n+1} \\
& \quad=\left[\frac{\lambda}{2 \pi\left[b(T)-b\left(t_{n}\right)\right]}\right]^{1 / 2} \int_{L_{2}[0, T]} \int_{\mathbb{R}} \exp \left\{-\frac{\lambda}{2\left[b(T)-b\left(t_{n}\right)\right]} \xi_{n+1}^{2}+i \xi_{n+1}\left\langle v \alpha_{n+1}, \alpha_{n+1}\right\rangle-\frac{1}{2 \lambda}\left\|\mathscr{P}^{\perp} M_{h} v\right\|^{2}\right. \\
& \left.\quad+i\left[\left(v, y+A+P_{b, n}\left(\vec{\xi}_{n}\right)\right)+\left[a(T)-a\left(t_{n}\right)\right]\left\langle v \alpha_{n+1}, \alpha_{n+1}\right\rangle\right]\right\} d \xi_{n+1} d \sigma(v) \\
& \quad=\int_{L_{2}[0, T]} \exp \left\{-\frac{1}{2 \lambda}\left[\left[b(T)-b\left(t_{n}\right)\right]\left\langle v \alpha_{n+1}, \alpha_{n+1}\right\rangle^{2}+\left\|\mathscr{P}^{\perp} M_{h} v\right\|^{2}\right]+i\left(v, y+a+P_{b, n}\left(\vec{\xi}_{n}-\vec{a}_{n}\right)\right)\right\} d \sigma(v) .
\end{aligned}
$$

By Morera's theorem and the dominated convergence theorem we have the theorem.

Theorem 19. Under the assumptions as given in Theorem 18, one has that for $P_{Z_{n}}$ a.e. $\vec{\xi}_{n}, \vec{\eta}_{n} \in \mathbb{R}^{n+1}$

$$
\begin{aligned}
& \| T_{\bar{\lambda}}\left[T_{\lambda}\left[F_{Z} \mid Z_{n}\right]\left(\cdot, \vec{\xi}_{n}\right) \mid Z_{n}\right]\left(\cdot, \vec{\eta}_{n}\right) \\
& \quad-F\left(\cdot+2 a+P_{b, n}\left(\vec{\xi}_{n}+\vec{\eta}_{n}-2 \vec{a}_{n}\right)\right) \|_{p}
\end{aligned}
$$

converges to 0 and for $w_{\varphi}$ a.e. $y \in C[0, T]$

$$
\begin{aligned}
& T_{\bar{\lambda}}\left[T_{\lambda}\left[F_{Z} \mid Z_{n}\right]\left(\cdot, \vec{\xi}_{n}\right) \mid Z_{n}\right]\left(y, \vec{\eta}_{n}\right) \\
& \quad \longrightarrow F\left(y+2 a+P_{b, n}\left(\vec{\xi}_{n}+\vec{\eta}_{n}-2 \vec{a}_{n}\right)\right),
\end{aligned}
$$

as $\lambda$ approaches -iq through $\mathbb{C}_{+}$.
Proof. By Theorem 18, $T_{\bar{\lambda}}\left[T_{\lambda}\left[F_{Z} \mid Z_{n}\right]\left(\cdot, \vec{\xi}_{n}\right) \mid Z_{n}\right]\left(y, \vec{\eta}_{n}\right)$ is well-defined so that we have for $\lambda \in \mathbb{C}_{+}$

$$
\begin{aligned}
T_{\bar{\lambda}} & {\left[T_{\lambda}\left[F_{Z} \mid Z_{n}\right]\left(\cdot, \vec{\xi}_{n}\right) \mid Z_{n}\right]\left(y, \vec{\eta}_{n}\right) } \\
& =\int_{L_{2}[0, T]} \exp \left\{-\frac{\operatorname{Re} \lambda}{|\lambda|^{2}}\left[\left[b(T)-b\left(t_{n}\right)\right]\left\langle v \alpha_{n+1}, \alpha_{n+1}\right\rangle^{2}\right.\right. \\
& \left.+\left\|\mathscr{P}^{\perp} M_{h} v\right\|^{2}\right] \\
& \left.+i\left[\left(v, y+2 a+P_{b, n}\left(\vec{\xi}_{n}+\vec{\eta}_{n}-2 \vec{a}_{n}\right)\right)\right]\right\} d \sigma(v) .
\end{aligned}
$$

Applying a similar method as used in the proof of Theorem 4.2 in [11] with minor modifications we can obtain the remainder part of the proof. 
Theorem 20. Let $F_{1}$ and $F_{2}$ and $\sigma_{1}$ and $\sigma_{2}$ be related by (60), respectively. Then for $\lambda \in \mathbb{C}_{+}, w_{\varphi}$ a.e. $y \in C[0, T]$, and a.e. $\vec{\xi}_{n} \in \mathbb{R}^{n+1}$

$$
\begin{aligned}
& {\left[\left(\left(F_{1}\right)_{Z} *\left(F_{2}\right)_{Z}\right)_{\lambda} \mid Z_{n}\right]\left(y, \vec{\xi}_{n}\right)} \\
& \quad=\int_{L_{2}[0, T]} \int_{L_{2}[0, T]} \exp \left\{-\frac{1}{4 \lambda}\left[\left[b(T)-b\left(t_{n}\right)\right]\left\langle\left(v_{1}-v_{2}\right) \alpha_{n+1}, \alpha_{n+1}\right\rangle^{2}+\left\|\mathscr{P}^{\perp} M_{h}\left(v_{1}-v_{2}\right)\right\|^{2}\right]\right. \\
& \left.\quad+\frac{i}{\sqrt{2}}\left[\left(v_{1}+v_{2}, y\right)+\left(v_{1}-v_{2}, a+P_{b, n}\left(\vec{\xi}_{n}-\vec{a}_{n}\right)\right)\right]\right\} d \sigma_{1}\left(v_{1}\right) d \sigma_{2}\left(v_{2}\right) .
\end{aligned}
$$

For a nonzero real $q,\left[\left(\left(F_{1}\right)_{Z} *\left(F_{2}\right)_{Z}\right)_{q} \mid Z_{n}\right]\left(y, \vec{\xi}_{n}\right)$ is given by the above equation, where $\lambda$ is replaced by-iq. Furthermore, $\left[\left(\left(F_{1}\right)_{Z} *\left(F_{2}\right)_{Z}\right)_{q} \mid Z_{n}\right]\left(\cdot, \vec{\xi}_{n}\right) \in \mathscr{A}^{(\infty)}$.

Proof. For $\vec{\xi}_{n}=\left(\xi_{0}, \xi_{1}, \ldots, \xi_{n}\right) \in \mathbb{R}^{n+1}$ let $\vec{\xi}_{n+1}=\left(\xi_{0}, \xi_{1}, \ldots\right.$, $\left.\xi_{n}, \xi_{n+1}\right)$, where $\xi_{n+1} \in \mathbb{R}$. Then for $\lambda>0, w_{\varphi}$ a.e. $y \in C[0, T]$, and $P_{Z_{n}}$ a.e. $\vec{\xi}_{n} \in \mathbb{R}^{n+1}$, we have by Lemmas 1 and 2

$$
\begin{aligned}
& K_{\left(F_{1}\right)_{Z},\left(F_{2}\right)_{Z}}^{\lambda}\left(y, \vec{\xi}_{n}\right) \\
& =\left[\frac{\lambda}{2 \pi\left[b(T)-b\left(t_{n}\right)\right]}\right]^{1 / 2} \\
& \cdot \int_{L_{2}[0, T]} \int_{L_{2}[0, T]} \int_{\mathbb{R}} \int_{C[0, T]} \exp \left\{-\frac{\lambda}{2\left[b(T)-b\left(t_{n}\right)\right]}\right. \\
& \cdot\left(\xi_{n+1}-\xi_{n}-\left[a(T)-a\left(t_{n}\right)\right]\right)^{2} \\
& +\frac{i}{\sqrt{2}}\left[\left(v_{1}, y+\lambda^{-1 / 2} X_{b, n+1}(x, \cdot)+A+P_{b, n+1}\left(\vec{\xi}_{n+1}\right)\right)\right. \\
& +\left(v_{2}, y-\lambda^{-1 / 2} X_{b, n+1}(x, \cdot)\right. \\
& \left.\left.\left.-A-P_{b, n+1}\left(\vec{\xi}_{n+1}\right)\right)\right]\right\} d w_{\varphi}(x) d \xi_{n+1} d \sigma_{1}\left(v_{1}\right) d \sigma_{2}\left(v_{2}\right) \\
& =\left[\frac{\lambda}{2 \pi\left[b(T)-b\left(t_{n}\right)\right]}\right]^{1 / 2} \\
& \cdot \int_{L_{2}[0, T]} \int_{L_{2}[0, T]} \int_{\mathbb{R}} \exp \left\{-\frac{\lambda \xi_{n+1}^{2}}{2\left[b(T)-b\left(t_{n}\right)\right]}\right. \\
& +\frac{i}{\sqrt{2}}\left[\left(v_{1}+v_{2}, y\right)+\left(v_{1}-v_{2}, A\right)+\left(v_{1}-v_{2}, P_{b, n}\left(\vec{\xi}_{n}\right)\right)\right. \\
& \left.+\left[a(T)-a\left(t_{n}\right)+\xi_{n+1}\right]\left\langle\left(v_{1}-v_{2}\right) \alpha_{n+1}, \alpha_{n+1}\right\rangle\right] \\
& \left.-\frac{1}{4 \lambda}\left\|\mathscr{P}^{\perp} M_{h}\left(v_{1}-v_{2}\right)\right\|^{2}\right\} d \xi_{n+1} d \sigma_{1}\left(v_{1}\right) d \sigma_{2}\left(v_{2}\right) \\
& =\int_{L_{2}[0, T]} \int_{L_{2}[0, T]} \exp \left\{-\frac{1}{4 \lambda}\left[\left[b(T)-b\left(t_{n}\right)\right]\right.\right. \\
& \left.\cdot\left\langle\left(v_{1}-v_{2}\right) \alpha_{n+1}, \alpha_{n+1}\right\rangle^{2}+\left\|\mathscr{P}^{\perp} M_{h}\left(v_{1}-v_{2}\right)\right\|^{2}\right] \\
& +\frac{i}{\sqrt{2}}\left[\left(v_{1}+v_{2}, y\right)\right. \\
& \left.\left.+\left(v_{1}-v_{2}, a+P_{b, n}\left(\vec{\xi}_{n}-\vec{a}_{n}\right)\right)\right]\right\} d \sigma_{1}\left(v_{1}\right) d \sigma_{2}\left(v_{2}\right) .
\end{aligned}
$$

By Morera's theorem and the dominated convergence theorem we have the theorem.

Theorem 21. Let $q$ be a nonzero real number and let $1 \leq p \leq$ $\infty$. Furthermore let $F_{1}$ and $F_{2}$ be as given in Theorem 20. Then one has for $w_{\varphi}$ a.e. $y \in C[0, T]$ and $P_{Z_{n}}$ a.e. $\vec{\xi}_{n}, \vec{\eta}_{n} \in \mathbb{R}^{n+1}$

$$
\begin{aligned}
T_{q}^{(p)} & {\left[\left[\left(\left(F_{1}\right)_{Z} *\left(F_{2}\right)_{Z}\right)_{q} \mid Z_{n}\right]\left(\cdot, \vec{\xi}_{n}\right) \mid Z_{n}\right]\left(y, \vec{\eta}_{n}\right) } \\
= & {\left[T _ { q } ^ { ( p ) } [ ( F _ { 1 } ) _ { Z } | Z _ { n } ] \left(\frac{1}{\sqrt{2}} y\right.\right.} \\
+ & \left.\left.(\sqrt{2}-1) a, \frac{1}{\sqrt{2}}\left(\vec{\eta}_{n}+\vec{\xi}_{n}\right)-(\sqrt{2}-1) \vec{a}_{n}\right)\right] \\
& \cdot\left[T _ { q } ^ { ( p ) } [ ( F _ { 2 } ) _ { Z } | Z _ { n } ] \left(\frac{1}{\sqrt{2}} y-a, \frac{1}{\sqrt{2}}\left(\vec{\eta}_{n}-\vec{\xi}_{n}\right)\right.\right. \\
& \left.\left.+\vec{a}_{n}\right)\right] .
\end{aligned}
$$

Proof. By Theorems 18 and 20 we have for $\lambda \in \mathbb{C}_{+}, w_{\varphi}$ a.e. $y \in C[0, T]$, and $P_{Z_{n}}$ a.e. $\vec{\xi}_{n}, \vec{\eta}_{n} \in \mathbb{R}^{n+1}$

$$
\begin{aligned}
T_{\lambda} & {\left[\left[\left(\left(F_{1}\right)_{Z} *\left(F_{2}\right)_{Z}\right)_{q} \mid Z_{n}\right]\left(\cdot, \vec{\xi}_{n}\right) \mid Z_{n}\right]\left(y, \vec{\eta}_{n}\right) } \\
= & \int_{L_{2}[0, T]} \int_{L_{2}[0, T]} \exp \left\{\frac { 1 } { 4 q i } \left[\left[b(T)-b\left(t_{n}\right)\right]\right.\right. \\
& \cdot\left\langle\left(v_{1}+v_{2}\right) \alpha_{n+1}, \alpha_{n+1}\right\rangle^{2} \\
& \left.+\left\|\mathscr{P}^{\perp} M_{h}\left(v_{1}+v_{2}\right)\right\|^{2}\right]-\frac{1}{4 \lambda}\left[\left[b(T)-b\left(t_{n}\right)\right]\right. \\
& \cdot\left\langle\left(v_{1}+v_{2}\right) \alpha_{n+1}, \alpha_{n+1}\right\rangle^{2} \\
& \left.+\left\|\mathscr{P}^{\perp} M_{h}\left(v_{1}+v_{2}\right)\right\|^{2}\right] \\
& +\frac{i}{\sqrt{2}}\left[\left(v_{1}+v_{2}, y+a+P_{b, n}\left(\vec{\eta}_{n}-\vec{a}_{n}\right)\right)\right. \\
& \left.\left.+\left(v_{1}-v_{2}, a+P_{b, n}\left(\vec{\xi}_{n}-\vec{a}_{n}\right)\right)\right]\right\} d \sigma_{1}\left(v_{1}\right) d \sigma_{2}\left(v_{2}\right) .
\end{aligned}
$$

Let $T_{q}^{(p)}\left[\left[\left(\left(F_{1}\right)_{Z} *\left(F_{2}\right)_{Z}\right)_{q} \mid Z_{n}\right]\left(\cdot, \vec{\xi}_{n}\right) \mid Z_{n}\right]\left(y, \vec{\eta}_{n}\right)$ be given by the right-hand side of the above equality, where $\lambda$ is replaced 
by -iq. The existence of $T_{q}^{(1)}\left[\left[\left(\left(F_{1}\right)_{Z} *\left(F_{2}\right)_{Z}\right)_{q} \mid Z_{n}\right]\left(\cdot, \vec{\xi}_{n}\right) \mid\right.$ $\left.Z_{n}\right]\left(y, \vec{\eta}_{n}\right)$ follows from the dominated convergence theorem. Now let $1<p \leq \infty$ and $1 / p+1 / p^{\prime}=1$. Then we have by the dominated convergence theorem

$$
\begin{aligned}
& \int_{C[0, T]} \mid T_{\lambda}\left[\left[\left(\left(F_{1}\right)_{Z} *\left(F_{2}\right)_{Z}\right)_{q} \mid Z_{n}\right]\left(\cdot, \vec{\xi}_{n}\right) \mid Z_{n}\right]\left(y, \vec{\eta}_{n}\right) \\
& -\left.T_{q}^{(p)}\left[\left[\left(\left(F_{1}\right)_{Z} *\left(F_{2}\right)_{Z}\right)_{q} \mid Z_{n}\right]\left(\cdot, \vec{\xi}_{n}\right) \mid Z_{n}\right]\left(y, \vec{\eta}_{n}\right)\right|^{p^{\prime}} d w_{\varphi}(y) \\
& \leq\left[\int_{L_{2}[0, T]} \int_{L_{2}[0, T]} \mid \exp \left\{-\frac{1}{4 \lambda}\left[\left[b(T)-b\left(t_{n}\right)\right]\right.\right.\right. \\
& \left.\left.\cdot\left\langle\left(v_{1}+v_{2}\right) \alpha_{n+1}, \alpha_{n+1}\right\rangle^{2}+\left\|\mathscr{P}^{\perp} M_{h}\left(v_{1}+v_{2}\right)\right\|^{2}\right]\right\} \\
& -\exp \left\{\frac { 1 } { 4 q i } \left[\left[b(T)-b\left(t_{n}\right)\right]\left\langle\left(v_{1}+v_{2}\right) \alpha_{n+1}, \alpha_{n+1}\right\rangle^{2}\right.\right. \\
& \left.\left.\left.\quad+\left\|\mathscr{P}^{\perp} M_{h}\left(v_{1}+v_{2}\right)\right\|^{2}\right]\right\}|d| \sigma_{1}\left|\left(v_{1}\right) d\right| \sigma_{2} \mid\left(v_{2}\right)\right]^{p^{\prime}} \longrightarrow 0,
\end{aligned}
$$

as $\lambda$ approaches -iq through $\mathbb{C}_{+}$, which shows the existence of $T_{q}^{(p)}\left[\left[\left(\left(F_{1}\right)_{Z} *\left(F_{2}\right)_{Z}\right)_{q} \mid Z_{n}\right]\left(\cdot, \vec{\xi}_{n}\right) \mid Z_{n}\right]\left(y, \vec{\eta}_{n}\right)$. The equality in the theorem follows from Theorem 18 with simple calculations.

Remark 22. (1) Theorems 15 and 17 can be obtained from Theorems 9 and 12, respectively. They also can be proved directly with aids of Theorems 14 and 16.

(2) Comparing Theorems 17 and 21 with Theorem 13, the results in Theorems 17 and 21 hold for $1 \leq p \leq \infty$.

\section{Change of Scale Formulas for the Transforms and Convolutions}

In this section, we derive two types of change of scale formulas for the conditional expectations as described in the previous sections.

For $\lambda \in \mathbb{C}, x \in C[0, T]$ and $\vec{\xi}_{n+1} \in \mathbb{R}^{n+2}$ let

$$
\begin{aligned}
K_{1}\left(\lambda, \vec{\xi}_{n+1}, x\right)=\left(\frac{\left|\Sigma_{M_{h}}\right|}{\left|\Sigma_{\mathscr{P} \perp}\right|}\right)^{1 / 2} \\
\cdot \exp \left\{\frac{1}{2}\left\|\Sigma_{M_{h}}^{-1 / 2}(\vec{v}, X(x, \cdot))\right\|_{\mathbb{R}}^{2}\right. \\
\left.-\frac{\lambda}{2}\left\|\Sigma_{\mathscr{P} \perp}^{-1 / 2}\left(\vec{v}, Z(x, \cdot)-A-P_{b, n+1}\left(\vec{\xi}_{n+1}\right)\right)\right\|_{\mathbb{R}}^{2}\right\} .
\end{aligned}
$$

For a nonzero real $q$, let $\left\{\lambda_{m}\right\}$ be any sequence in $\mathbb{C}_{+}$ converging to $-i q$.

By simple calculations, we have the following two theorems.
Theorem 23. Let $1 \leq p \leq \infty$ and let $F \in \mathscr{A}^{(p)}$. Then for $\lambda \in \mathbb{C}_{+}$, $w_{\varphi}$ a.e. $y \in C[0, T]$, and $P_{Z_{n}}$ a.e. $\vec{\xi}_{n}=\left(\xi_{0}, \xi_{1}, \ldots, \xi_{n}\right) \in$ $\mathbb{R}^{n+1}$

$$
\begin{gathered}
T_{\lambda}\left[F_{Z} \mid Z_{n}\right]\left(y, \vec{\xi}_{n}\right)=\lambda^{r / 2} \int_{\mathbb{R}} \Psi_{1}(\lambda, a(T) \\
\left.-a\left(t_{n}\right), b(T)-b\left(t_{n}\right), \xi_{n+1}-\xi_{n}\right) \\
\cdot E\left[K_{1}\left(\lambda, \vec{\xi}_{n+1}, \cdot\right) F_{Z}(\cdot, y)\right] d \xi_{n+1},
\end{gathered}
$$

where $\vec{\xi}_{n+1}=\left(\xi_{0}, \xi_{1}, \ldots, \xi_{n}, \xi_{n+1}\right)$ and $K_{1}$ is given by (72). If $p=$ 1 , then

$$
\begin{aligned}
T_{q}^{(1)} & {\left[F_{Z} \mid Z_{n}\right]\left(y, \vec{\xi}_{n}\right)=\lim _{m \rightarrow \infty} \lambda_{m}^{r / 2} \int_{\mathbb{R}} \Psi_{1}\left(\lambda_{m}, a(T)\right.} \\
- & \left.a\left(t_{n}\right), b(T)-b\left(t_{n}\right), \xi_{n+1}-\xi_{n}\right) \\
\cdot & E\left[K_{1}\left(\lambda_{m}, \vec{\xi}_{n+1}, \cdot\right) F_{Z}(\cdot, y)\right] d \xi_{n+1} .
\end{aligned}
$$

Theorem 24. Let $F \in \mathscr{A}^{\left(p_{1}\right)}$ and $G \in \mathscr{A}^{\left(p_{2}\right)}$ with $1 \leq p_{1}, p_{2} \leq$ $\infty$. Then for $\lambda \in \mathbb{C}_{+}, w_{\varphi}$ a.e. $y \in C[0, T]$, and $P_{Z_{n}}$ a.e. $\vec{\xi}_{n} \in$ $\mathbb{R}^{n+1},\left[\left(F_{Z} * G_{Z}\right)_{\lambda} \mid Z_{n}\right]\left(y, \vec{\xi}_{n}\right)$ is given by the right-hand side of (73) with replacing $F_{Z}(\cdot, y)$ by $F_{Z}(\cdot / \sqrt{2}, y / \sqrt{2}) G_{Z}(-\cdot / \sqrt{2}$, $y / \sqrt{2})$. Moreover if $p_{1}=p_{2}=1$, then $\left[\left(F_{Z} * G_{Z}\right)_{q} \mid Z_{n}\right]\left(y, \vec{\xi}_{n}\right)$ is given by the right-hand side of (74) with replacing $F_{Z}(\cdot, y)$ by $F_{Z}(\cdot / \sqrt{2}, y / \sqrt{2}) G_{Z}(-\cdot / \sqrt{2}, y / \sqrt{2})$.

By Theorems 14 and 23 and the dominated convergence theorem, we have the following theorem.

Theorem 25. Let $1 \leq p \leq \infty$ and let $\Pi$ be given by (54). Then, for $\lambda \in \mathbb{C}_{+}, w_{\varphi}$ a.e. $y \in C[0, T]$, and $P_{Z_{n}}$ a.e. $\vec{\xi}_{n} \in \mathbb{R}^{n+1}$, $T_{\lambda}\left[\Pi_{Z} \mid Z_{n}\right]\left(y, \vec{\xi}_{n}\right)$ and $T_{q}^{(p)}\left[\Pi_{Z} \mid Z_{n}\right]\left(y, \vec{\xi}_{n}\right)$ are given by the right-hand sides of (73) and (74), respectively, with replacing $F_{Z}$ by $\Pi_{Z}$.

By Theorems 16 and 24 and the dominated convergence theorem, we have the following theorem.

Theorem 26. Let the assumptions be as given in Theorem 16. Then, for $\lambda \in \mathbb{C}_{+}, w_{\varphi}$ a.e. $y \in C[0, T]$, and $P_{Z_{n}}$ a.e. $\vec{\xi}_{n} \in \mathbb{R}^{n+1}$, $\left[\left(\left(\Pi_{1}\right)_{Z} *\left(\Pi_{2}\right)_{Z}\right)_{\lambda} \mid Z_{n}\right]\left(y, \vec{\xi}_{n}\right)$ and $\left[\left(\left(\Pi_{1}\right)_{Z} *\left(\Pi_{2}\right)_{Z}\right)_{q} \mid Z_{n}\right](y$, $\left.\vec{\xi}_{n}\right)$ are given by the right-hand sides of (73) and (74), respectively, with replacing $F_{Z}$ by $\left(\Pi_{1}\right)_{Z}(\cdot / \sqrt{2}, y / \sqrt{2})\left(\Pi_{2}\right)_{Z}(-\cdot / \sqrt{2}$, $y / \sqrt{2})$.

For $\lambda \in \mathbb{C}, x \in C[0, T]$ and $\vec{\xi}_{n} \in \mathbb{R}^{n+1}$ let

$$
\begin{aligned}
K_{2}\left(\lambda, \vec{\xi}_{n}, x\right)=\left[\frac{\left|\Sigma_{M_{h}}\right|}{\Lambda\left(\Sigma_{\mathscr{P} \perp}\right)\left|\Sigma_{\mathscr{P} \perp}\right|}\right]^{1 / 2} \\
\cdot \exp \left\{\frac{1}{2}\left\|\Sigma_{M_{h}}^{-1 / 2}(\vec{v}, X(x, \cdot))\right\|_{\mathbb{R}}^{2}\right. \\
-\frac{\lambda}{2}\left\|\Sigma_{\mathscr{P} \perp}^{-1 / 2}\left(\vec{v}, Z(x, \cdot)-a-P_{b, n}\left(\vec{\xi}_{n}-\vec{a}_{n}\right)\right)\right\|_{\mathbb{R}}^{2}
\end{aligned}
$$




$$
\begin{aligned}
& +\frac{\lambda}{2 \Lambda\left(\Sigma_{\mathscr{P}^{\perp}}\right)}\left[b(T)-b\left(t_{n}\right)\right] \\
& \cdot\left\langle\Sigma_{\mathscr{P}^{\perp}}^{-1}\left(\vec{v}, Z(x, \cdot)-a-P_{b, n}\left(\vec{\xi}_{n}-\vec{a}_{n}\right)\right),\right. \\
& \left.\left.\left\langle\vec{v} \alpha_{n+1}, \alpha_{n+1}\right\rangle\right\rangle_{\mathbb{R}}^{2}\right\} .
\end{aligned}
$$

Theorem 27. Let $1 \leq p \leq \infty$ and let $F\left(\in \mathscr{A}^{(p)}\right)$ be given by (29). Then for $\lambda \in \mathbb{C}_{+}, w_{\varphi}$ a.e. $y \in C[0, T]$, and $P_{Z_{n}}$ a.e. $\vec{\xi}_{n} \in \mathbb{R}^{n+1}$

$$
T_{\lambda}\left[F_{Z} \mid Z_{n}\right]\left(y, \vec{\xi}_{n}\right)=\lambda^{r / 2} E\left[K_{2}\left(\lambda, \vec{\xi}_{n}, \cdot\right) F_{Z}(\cdot, y)\right] \text {, }
$$

where $K_{2}$ is given by (75). If $p=1$, then

$$
\begin{aligned}
T_{q}^{(1)} & {\left[F_{Z} \mid Z_{n}\right]\left(y, \vec{\xi}_{n}\right) } \\
\quad= & \lim _{m \rightarrow \infty} \lambda_{m}^{r / 2} E\left[K_{2}\left(\lambda_{m}, \vec{\xi}_{n}, \cdot\right) F_{Z}(\cdot, y)\right] .
\end{aligned}
$$

Proof. For $\lambda>0, w_{\varphi}$ a.e. $y \in C[0, T]$, and $P_{Z_{n}}$ a.e. $\vec{\xi}_{n} \in \mathbb{R}^{n+1}$, we have by Lemma 3

$$
\begin{aligned}
& \lambda^{r / 2} E\left[K_{2}\left(\lambda, \vec{\xi}_{n}, \cdot\right) F_{Z}(\cdot, y)\right]=\left[\frac{\lambda^{r}\left|\Sigma_{M_{h}}\right|}{\Lambda\left(\Sigma_{\mathscr{P} \perp}\right)\left|\Sigma_{\mathscr{P} \perp}\right|}\right]^{1 / 2} \int_{C[0, T]} F(Z(x, \cdot)+y) \exp \left\{\frac{1}{2}\left\|\Sigma_{M_{h}}^{-1 / 2}(\vec{v}, Z(x, \cdot)-a)\right\|_{\mathbb{R}}^{2}\right. \\
& \quad-\frac{\lambda}{2}\left\|\Sigma_{\mathscr{P}^{\perp}}^{-1 / 2}\left(\vec{v}, Z(x, \cdot)-a-P_{b, n}\left(\vec{\xi}_{n}-\vec{a}_{n}\right)\right)\right\|_{\mathbb{R}}^{2} \\
& \left.+\frac{\lambda}{2 \Lambda\left(\Sigma_{\mathscr{P} \perp}\right)}\left[b(T)-b\left(t_{n}\right)\right]\left\langle\Sigma_{\mathscr{P}^{\perp}}^{-1}\left(\vec{v}, Z(x, \cdot)-a-P_{b, n}\left(\vec{\xi}_{n}-\vec{a}_{n}\right)\right),\left\langle\vec{v} \alpha_{n+1}, \alpha_{n+1}\right\rangle\right\rangle_{\mathbb{R}}^{2}\right\} d w_{\varphi}(x) \\
& =\left[\frac{\lambda^{r}}{(2 \pi)^{r} \Lambda\left(\Sigma_{\mathscr{P} \perp}\right)\left|\Sigma_{\mathscr{P}^{\perp}}\right|}\right]^{1 / 2} \int_{\mathbb{R}^{r}} f(\vec{u}+(\vec{v}, y)) \exp \left\{-\frac{\lambda}{2}\left\|\Sigma_{\mathscr{P}^{\perp}}^{-1 / 2}\left(\vec{u}-\left(\vec{v}, a+P_{b, n}\left(\vec{\xi}_{n}-\vec{a}_{n}\right)\right)\right)\right\|_{\mathbb{R}}^{2}\right. \\
& \left.+\frac{\lambda}{2 \Lambda\left(\Sigma_{\mathscr{P} \perp}\right)}\left[b(T)-b\left(t_{n}\right)\right]\left\langle\Sigma_{\mathscr{P}^{\perp}}^{-1 / 2}\left(\vec{u}-\left(\vec{v}, a+P_{b, n}\left(\vec{\xi}_{n}-\vec{a}_{n}\right)\right)\right), \Sigma_{\mathscr{P}^{\perp}}^{-1 / 2}\left\langle\vec{v} \alpha_{n+1}, \alpha_{n+1}\right\rangle\right\rangle_{\mathbb{R}}^{2}\right\} d \vec{u} \\
& \quad=\left[\frac{\lambda^{r}}{(2 \pi)^{r} \Lambda\left(\Sigma_{\mathscr{P} \perp}\right)}\right]^{1 / 2} \int_{\mathbb{R}^{r}} f\left(\Sigma_{\mathscr{P} \perp}^{1 / 2} \vec{u}+\left(\vec{v}, y+a+P_{b, n}\left(\vec{\xi}_{n}-\vec{a}_{n}\right)\right)\right) \exp \left\{-\frac{\lambda}{2}\|\vec{u}\|_{\mathbb{R}}^{2}\right. \\
& \left.\quad+\frac{\lambda}{2 \Lambda\left(\Sigma_{\mathscr{P} \perp}\right)}\left[b(T)-b\left(t_{n}\right)\right]\left\langle\vec{u}, \Sigma_{\mathscr{P} \perp}^{-1 / 2}\left\langle\vec{v} \alpha_{n+1}, \alpha_{n+1}\right\rangle\right\rangle_{\mathbb{R}}^{2}\right\} d \vec{u} .
\end{aligned}
$$

By the analytic continuation, the dominated convergence theorem, and Theorem 5, we have the theorem.

Theorem 28. Let $F \in \mathscr{A}^{\left(p_{1}\right)}$ and $G \in \mathscr{A}^{\left(p_{2}\right)}$ with $1 \leq p_{1}, p_{2} \leq$ $\infty$. Then for $\lambda \in \mathbb{C}_{+}, w_{\varphi}$ a.e. $y \in C[0, T]$, and $P_{Z_{n}}$ a.e. $\vec{\xi}_{n} \in$ $\mathbb{R}^{n+1},\left[\left(F_{Z} * G_{Z}\right)_{\lambda} \mid Z_{n}\right]\left(y, \vec{\xi}_{n}\right)$ is given by the right-hand side of (76) with replacing $F_{Z}(\cdot, y)$ by $F_{Z}(\cdot / \sqrt{2}, y / \sqrt{2}) G_{Z}(-\cdot / \sqrt{2}$, $y / \sqrt{2})$. Moreover if $p_{1}=p_{2}=1$, then $\left[\left(F_{Z} * G_{Z}\right)_{q} \mid Z_{n}\right]\left(y, \vec{\xi}_{n}\right)$ is given by the right-hand side of $(77)$ with replacing $F_{Z}(\cdot, y)$ by $F_{Z}(\cdot / \sqrt{2}, y / \sqrt{2}) G_{Z}(-\cdot / \sqrt{2}, y / \sqrt{2})$.

By Theorems 14, 16, and 27 and the dominated convergence theorem, we have the following two theorems.

Theorem 29. Let $1 \leq p \leq \infty$ and let $\Pi$ be given by (54). Then, for $\lambda \in \mathbb{C}_{+}, w_{\varphi}$ a.e. $y \in C[0, T]$, and $P_{Z_{n}}$ a.e. $\vec{\xi}_{n} \in \mathbb{R}^{n+1}$, $T_{\lambda}\left[\Pi_{Z} \mid Z_{n}\right]\left(y, \vec{\xi}_{n}\right)$ and $T_{q}^{(p)}\left[\Pi_{Z} \mid Z_{n}\right]\left(y, \vec{\xi}_{n}\right)$ are given by the right-hand sides of (76) and (77), respectively, with replacing $F_{Z}$ by $\Pi_{Z}$.
Theorem 30. Let the assumptions be as given in Theorem 16. Then, for $\lambda \in \mathbb{C}_{+}, w_{\varphi}$ a.e. $y \in C[0, T]$, and $P_{Z_{n}}$ a.e. $\vec{\xi}_{n} \in \mathbb{R}^{n+1}$, $\left[\left(\left(\Pi_{1}\right)_{Z} *\left(\Pi_{2}\right)_{Z}\right)_{\lambda} \mid Z_{n}\right]\left(y, \vec{\xi}_{n}\right)$ and $\left[\left(\left(\Pi_{1}\right)_{Z} *\left(\Pi_{2}\right)_{Z}\right)_{q} \mid Z_{n}\right](y$, $\left.\vec{\xi}_{n}\right)$ are given by the right-hand sides of (76) and (77), respectively, with replacing $F_{Z}$ by $\left(\Pi_{1}\right)_{Z}(\cdot / \sqrt{2}, y / \sqrt{2})\left(\Pi_{2}\right)_{Z}(-\cdot / \sqrt{2}$, $y / \sqrt{2})$.

Let $\left\{e_{1}, e_{2}, \ldots\right\}$ be a complete orthonormal basis of $L_{2}[0, T]$. For $m \in \mathbb{N}, \lambda \in \mathbb{C}$ and $x \in C[0, T]$ let

$$
H_{m}(\lambda, x)=\exp \left\{\frac{1-\lambda}{2} \sum_{j=1}^{m}\left(e_{j}, x\right)^{2}\right\}
$$

We now have the following relationships among the conditional Fourier-Feynman transform, the conditional convolution products, and the generalized Wiener integrals of the functions in $\mathcal{S}_{w_{\varphi}}$. Their proofs are similar to the proofs of the results in [12] with additional calculations. 
Theorem 31. Let $1 \leq p \leq \infty$ and let $F$ be given by (60). Then for $\lambda \in \mathbb{C}_{+}, w_{\varphi}$ a.e. $x \in C[0, T]$, and $P_{Z_{n}}$ a.e. $\vec{\xi}_{n}=\left(\xi_{0}, \xi_{1}, \ldots\right.$, $\left.\xi_{n}\right) \in \mathbb{R}^{n+1}$

$$
\begin{gathered}
T_{\lambda}\left[F_{Z} \mid Z_{n}\right]\left(y, \vec{\xi}_{n}\right)=\lim _{m \rightarrow \infty} \lambda^{m / 2} \int_{\mathbb{R}} \Psi_{1}(\lambda, a(T) \\
\left.-a\left(t_{n}\right), b(T)-b\left(t_{n}\right), \xi_{n+1}-\xi_{n}\right) E\left[H_{m}(\lambda, x)\right. \\
\left.\cdot F\left(y+Z_{b, n+1}(x, \cdot)+P_{b, n+1}\left(\vec{\xi}_{n+1}\right)\right)\right] d \xi_{n+1},
\end{gathered}
$$

where $\vec{\xi}_{n+1}=\left(\xi_{0}, \ldots, \xi_{n}, \xi_{n+1}\right)$ for $\xi_{n+1} \in \mathbb{R}$. Moreover, $T_{q}^{(p)}\left[F_{Z} \mid Z_{n}\right]\left(y, \vec{\xi}_{n}\right)$ is given by the right-hand side of the above equality with replacing $\lambda$ by $\lambda_{m}$.

Theorem 32. Let $F_{1}, F_{2}$, and $\sigma_{1}, \sigma_{2}$ be related by (60), respectively. Then for $\lambda \in \mathbb{C}_{+}, w_{\varphi}$ a.e. $y \in C[0, T]$, and $P_{Z_{n}}$ a.e. $\vec{\xi}_{n} \in$ $\mathbb{R}^{n+1}$

$$
\begin{aligned}
& {\left[\left(\left(F_{1}\right)_{Z} *\left(F_{2}\right)_{Z}\right)_{\lambda} \mid Z_{n}\right]\left(y, \vec{\xi}_{n}\right)=\lim _{m \rightarrow \infty} \lambda^{m / 2} \int_{\mathbb{R}} \Psi_{1}(\lambda, a(T)} \\
& \left.\quad-a\left(t_{n}\right), b(T)-b\left(t_{n}\right), \xi_{n+1}-\xi_{n}\right) E\left[H_{m}(\lambda, x)\right. \\
& \quad \cdot F_{1}\left(\frac{1}{\sqrt{2}}\left[y+Z_{b, n+1}(x, \cdot)+P_{b, n+1}\left(\vec{\xi}_{n+1}\right)\right]\right) \\
& \left.\cdot F_{2}\left(\frac{1}{\sqrt{2}}\left[y-Z_{b, n+1}(x, \cdot)-P_{b, n+1}\left(\vec{\xi}_{n+1}\right)\right]\right)\right] d \xi_{n+1} .
\end{aligned}
$$

Moreover, $\left[\left(\left(F_{1}\right)_{Z} *\left(F_{2}\right)_{Z}\right)_{q} \mid Z_{n}\right]\left(y, \vec{\xi}_{n}\right)$ is given by the righthand side of the above equality with replacing $\lambda$ by $\lambda_{m}$.

Remark 33. Letting $\lambda=\gamma^{-2}$ in the theorems of this section, where $\gamma>0$, we can obtain the change of scales for $E\left[F_{Z}(\gamma \cdot\right.$, y) $\left.\mid Z_{n}(\gamma \cdot)\right]$ and $E\left[F_{Z}(\gamma \cdot / \sqrt{2}, y / \sqrt{2}) G_{Z}(-\gamma \cdot / \sqrt{2}, y / \sqrt{2}) \mid\right.$ $\left.Z_{n}(\gamma \cdot)\right]$; that is, the scale $\gamma$ in the functions $F_{Z}(\gamma \cdot y), G_{Z}(-\gamma \cdot /$ $\sqrt{2}, y / \sqrt{2})$, and $Z_{n}(\gamma \cdot)$ is moved to $K_{1}, K_{2}, \Psi_{1}$, and $H_{m}$ in evaluating the conditional expectations as above.

Remark 34. (1) An orthonormal subset $\left\{v_{1}, v_{2}, \ldots, v_{r}\right\}$ of $L_{2}[0, T]$ such that both $\left\{M_{h} v_{1}, \ldots, M_{h} v_{r}\right\}$ and $\left\{\mathscr{P}^{\perp} M_{h} v_{1}\right.$, $\left.\ldots, \mathscr{P}^{\perp} M_{h} v_{r}\right\}$ are independent sets exists [12].

(2) Let $\left\{e_{11}, \ldots, e_{1 r}\right\}$ and $\left\{e_{21}, \ldots, e_{2 r}\right\}$ be the orthonormal sets obtained from $\left\{M_{h} v_{1}, \ldots, M_{h} v_{r}\right\}$ and $\left\{\mathscr{P}^{\perp} M_{h} v_{1}\right.$, $\left.\ldots, \mathscr{P}^{\perp} M_{h} v_{r}\right\}$, respectively, by the Gram-Schmidt orthonormalization process. For $l=1, \ldots, r$, let

$$
\begin{array}{r}
M_{h} v_{l}=\sum_{j=1}^{r} \alpha_{l j} e_{1 j}, \\
\mathscr{P}^{\perp} M_{h} v_{l}=\sum_{j=1}^{r} \beta_{l j} e_{2 j}
\end{array}
$$

be the linear combinations. Let $B_{1}=\left[\alpha_{l j}\right]_{r \times r}$ and $B_{2}=\left[\beta_{l j}\right]_{r \times r}$ be the coefficient matrices of the combinations. Then $M_{h}^{1 / 2}$ and $\Sigma_{\mathscr{P} \perp}^{1 / 2}$ can be replaced by $B_{1}$ and $B_{2}$, respectively, in each expression of the theorems.
(3) It does not mean that $B_{1}=\Sigma_{M_{h}}^{1 / 2}$ or $B_{2}=\Sigma_{\mathscr{P}^{\perp}}^{1 / 2}$ in (2). They only satisfy the following equations:

$$
\begin{aligned}
& B_{1} B_{1}^{T}=\Sigma_{M_{h}}=\left(\Sigma_{M_{h}}^{1 / 2}\right)^{2}, \\
& B_{2} B_{2}^{T}=\Sigma_{\mathscr{P}^{\perp}}=\left(\Sigma_{\mathscr{P} \perp}^{1 / 2}\right)^{2} .
\end{aligned}
$$

Remark 35. (1) If $y=0$, then we can obtain the change of scale formulas in [2] with $Z_{n}$.

(2) If $a=0$ and $y=0$, then we can obtain the results in [12] with the cylinder functions.

(3) The results of this paper are independent of a particular choice of the initial distribution $\varphi$.

\section{Competing Interests}

The author declares that they have no competing interests.

\section{Acknowledgments}

This work was supported by Kyonggi University Research Grant 2015.

\section{References}

[1] M. K. Im and K. S. Ryu, "An analogue of Wiener measure and its applications," Journal of the Korean Mathematical Society, vol. 39, no. 5, pp. 801-819, 2002.

[2] D. H. Cho, "Integral transforms on a function space with change of scales using multivariate normal distributions," Journal of Function Spaces, vol. 2016, Article ID 9235960, 9 pages, 2016.

[3] R. H. Cameron, "The translation pathology of Wiener space," Duke Mathematical Journal, vol. 21, pp. 623-627, 1954.

[4] R. H. Cameron and W. T. Martin, "The behavior of measure and measurability under change of scale in Wiener space," Bulletin of the American Mathematical Society, vol. 53, pp. 130-137, 1947.

[5] D. H. Cho, "A conditional Fourier-Feynman transform and conditional convolution product with change of scales on a function space I," Bulletin of the Korean Mathematical Society, In press.

[6] D. H. Cho, "Analogues of conditional Wiener integrals with drift and initial distribution on a function space," Abstract and Applied Analysis, vol. 2014, Article ID 916423, 12 pages, 2014.

[7] D. H. Cho, B. J. Kim, and I. Yoo, "Analogues of conditional Wiener integrals and their change of scale transformations on a function space," Journal of Mathematical Analysis and Applications, vol. 359, no. 2, pp. 421-438, 2009.

[8] R. G. Laha and V. K. Rohatgi, Probability Theory, John Wiley \& Sons, New York, NY, USA, 1979.

[9] G. B. Folland, Real analysis, Pure and Applied Mathematics, John Wiley \& Sons, New York, 1984.

[10] G. W. Johnson and D. L. Skoug, "The Cameron-Storvick function space integral: an $\mathscr{L}\left(L_{p}, L_{p^{\prime}}\right)$ theory," Nagoya Mathematical Journal, vol. 60, pp. 93-137, 1976.

[11] D. H. Cho, S. B. Park, and M. Go, "A present positiondependent conditional Fourier-Feynman Transform and convolution product over continuous paths," International Journal of Mathematical Analysis, vol. 9, no. 48, pp. 2387-2406, 2015.

[12] D. H. Cho and I. Yoo, "Change of scale formulas for a generalized conditional Wiener integral," Bulletin of the Korean Mathematical Society, vol. 53, no. 5, pp. 1531-1548, 2016. 


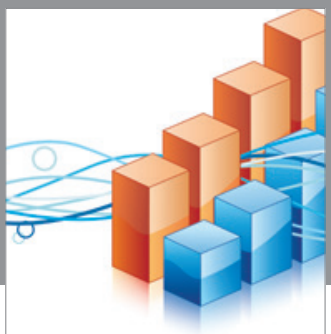

Advances in

Operations Research

vatem alat4

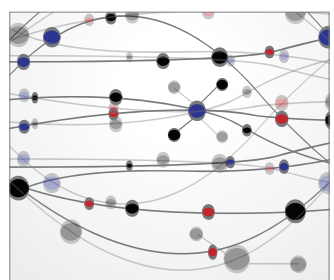

\section{The Scientific} World Journal
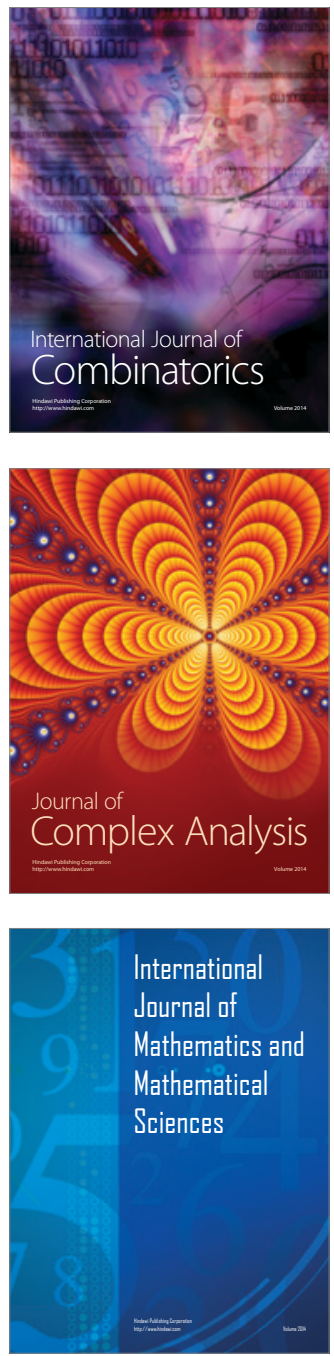
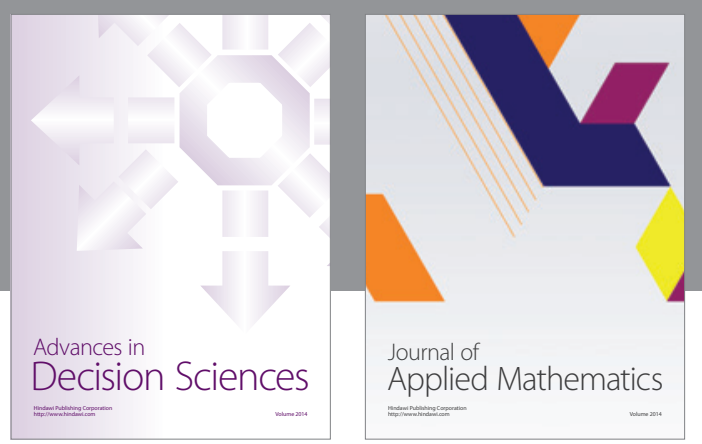

Algebra

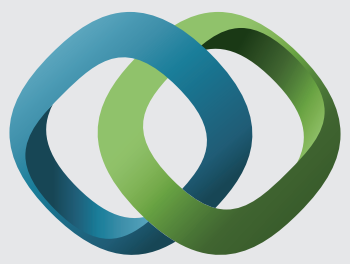

\section{Hindawi}

Submit your manuscripts at

https://www.hindawi.com
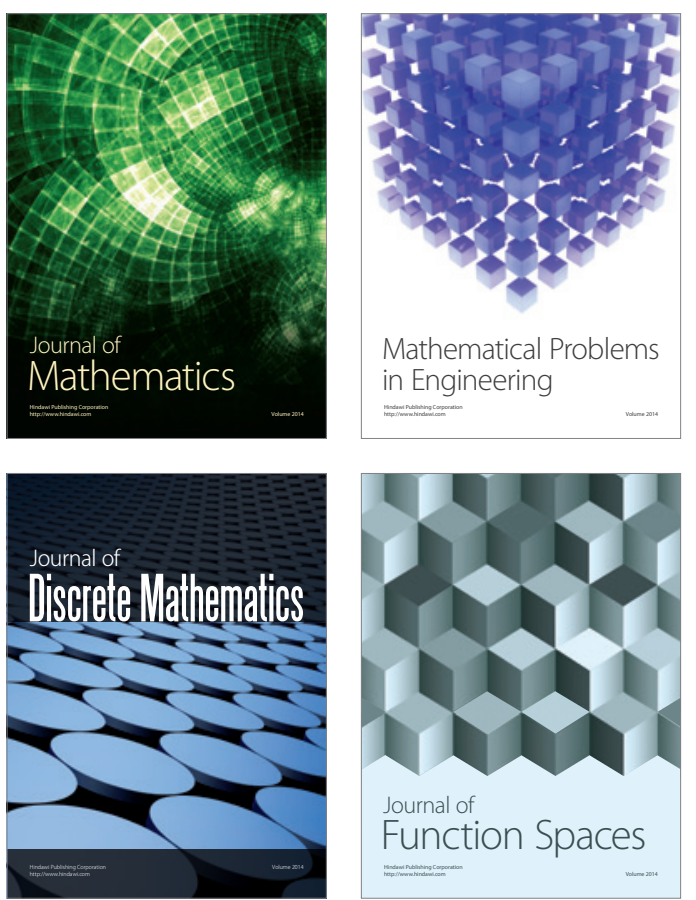

Mathematical Problems in Engineering
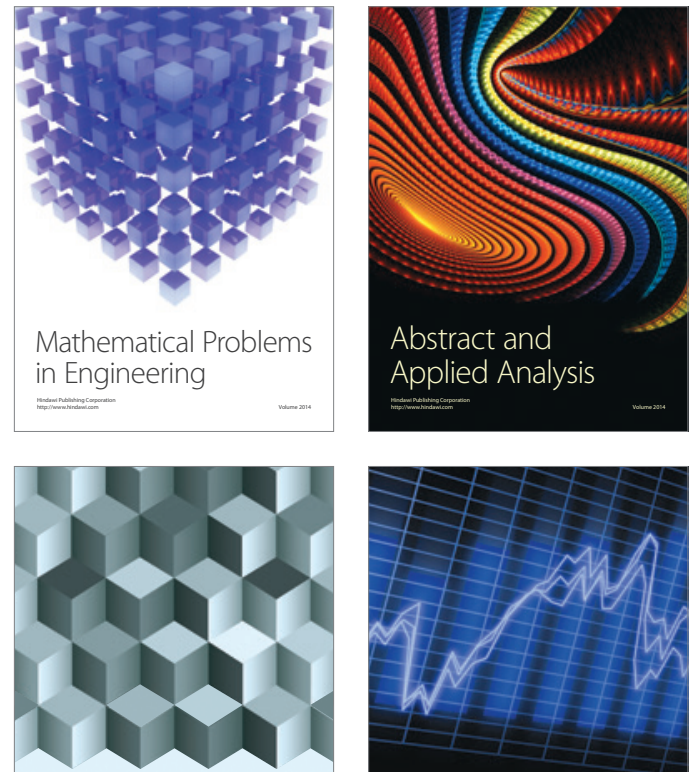

Journal of

Function Spaces

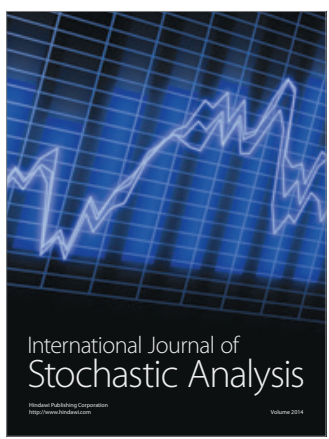

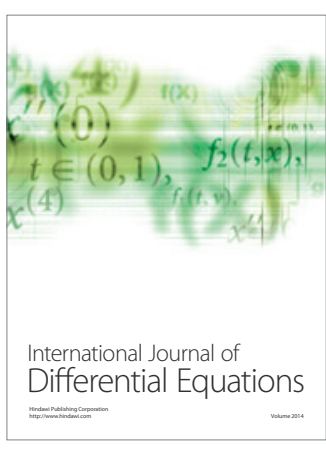
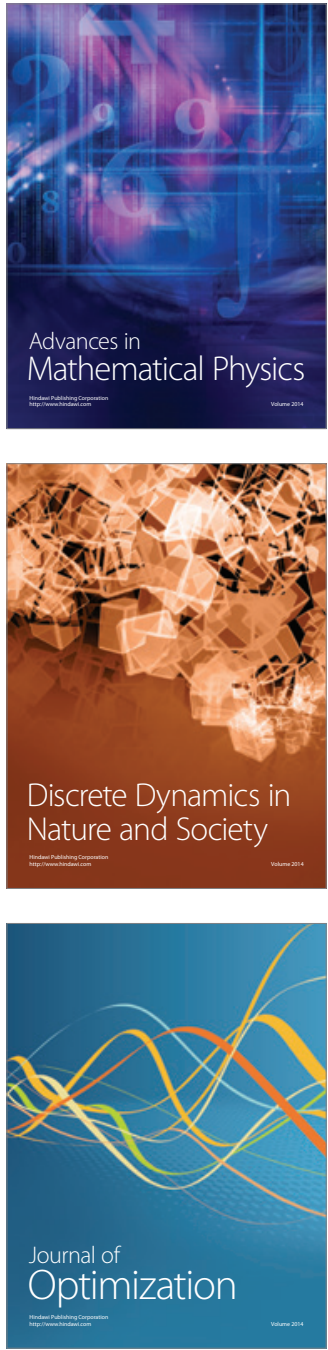